\title{
A Critical Edition and Philological Analysis of the Text of Isa 44:6-45:25 Based on the Coptic Manuscript sa 52 (M 568), Other Manuscripts Written in the Sahidic Coptic Dialect, and on the Greek Text of the Septuagint
}

\author{
TOMASZ BAKK
}

The John Paul II Catholic University of Lublin

tomciobak@gmail.com, ORCID: 0000-0003-0328-0282

\begin{abstract}
This paper contains a critical edition and philological analysis of Isa 44:6-45:25, which were worked out primarily on the basis of the Coptic manuscript sa 52.2 and other available manuscripts of the Sahidic dialect. Particular attention is paid to these verses since they occur only in manuscript sa 52.2 and so far have never been published. The first part presents general information on the fragment of codex sa 52 ( $\mathrm{M}$ 568) that includes the discussed text. The next part provides a list and brief characteristics of the other manuscripts containing at least some verses of Isa 44:6-45:25. The focal section of the paper is a presentation of the Coptic text (in the Sahidic dialect) and its translation into English. The differences between the Sahidic text and the Greek text of the Septuagint, on which the Coptic translation is based, have been pointed out in tables. They include additions and omissions in the Coptic translation, lexical changes, and semantic differences. Finally, the paper is devoted to difficult philological questions observed in the Coptic text itself or in its references to the Greek text of the LXX.
\end{abstract}

Keywords: Coptic, Sahidic dialect, the Book of Isaiah, Deutero-Isaiah, sa 52 (M 568), CLM 205, edition of Isa 44:6-45:25

After announcing Israel's liberation (Isa 42:8-44:5) the text of Deutero-Isaiah praises the magnitude of the true God (44:6-23). It stresses that the Lord - Israel's king is the first and the last, and there is no other God besides him (44:6-8), while all expressions of idolatry are nothing and useless (44:9-17). Facing the thoughtlessness of those who worship idols (44:18-20), the nation of Israel is called to faithfulness that brings joy (44:21-23).

The author of Deutero-Isaiah announces in a very concrete way that the deliverance of Israel will come through the Persian King Cyrus (Isa 44:24-45:13). This salvation will have not only a military but also spiritual significance. That is why, in the final part of our text there is a prophecy about the conversion and homage of the heathen (Isa 45:14-25). Foreign peoples will serve the true God (45:14-17), and God will address his message to all nations (45:18-25). 
The edition of the Coptic fragment of Isa 44:6-45:25 is a continuation of the earlier chapters of the Book of Isaiah that has been analysed so far. ${ }^{1}$ It will mainly be based on the Sahidic manuscript sa 52 (M 568), ${ }^{2}$ which is registered as CLM 205 in the contemporary electronic database. ${ }^{3}$ The present work is built both on the photographic edition (facsimile) and the microfilm made accessible to me by the Morgan Library in New York. For the last few years, the black and white photographs of this Coptic collection housed in the Library have been made available at https://archive. org/details/PhantoouLibrary. ${ }^{4}$

Apart from manuscript sa 52 belonging to the Pierpont Morgan Collection, I have considered the editions of all available Coptic manuscripts written in the Sahidic dialect that contain at least some verses of Isa 44:6-45:25. The critical edition and philological analysis of the selected text will be conducted in the following order: 1) general characteristics of the folios of the manuscript from the Pierpont Morgan collection, containing the analysed verses, 2) presentation of the Coptic text based on sa 52, including other available witnesses, 3) translation into English, 4) list of differences between the Greek text of the LXX and its Coptic translation, and 5) analysis of the more difficult philological phenomena appearing in the Coptic text.

\section{General Information on the Fragment of Manuscript sa 52}

The text of Isa 44:6 begins in line 30 of the left-hand column on the page designated $90 \mathrm{M} 568 \mathrm{f.} 44^{\mathrm{v}}$ in the facsimile edition. This page also has the Coptic number $\overline{\mathbf{4 B}}$, i.e. 92. The subsequent pages bear other Coptic signs, regularly placed in the upper outer edge. The only exception in the facsimile edition is the page marked as $94 \mathrm{M}$ $568 \mathrm{f} .46^{\mathrm{v}}$, which on both sides of its columns has two identical Coptic numbers $\overline{45}$. The number at the left-hand column was written in a definitely more thorough way, while the number on the right betrays a slightly different hand. So, it could have been written later. The five-dot punctuation was used next to this Coptic number.

1 The analysis of the text of Proto-Isaiah on the basis of manuscript sa 52 was the theme of the doctoral dissertation, submitted for publication. Its fragments are available in Italian: Bąk, Il Proto-Isaia in copto-saidico. The text of Isa $\mathbf{4 0}$ has been presented in: Bąk, Isa 40. The text of Isa 41 is available in: Bąk, Isa 41. The text of Isa 42:1-44:5 has been presented in: Bąk, Isa 42:1-44:5.

2 The history and general description of the manuscript in Bąk, Proto-Isaia, 13-28. A description of the whole Pierpont Morgan collection, containing sa 52 (marked by Depuydt as M 568), in Depuydt, Catalogue of Coptic Manuscripts, 20-22.

3 See https://atlas.paths-erc.eu/manuscripts/205 [access: 25.12.2019].

4 Isa 44:6 begins at https://archive.org/stream/PhantoouLibrary/m568\%20Combined\%20\%28Bookmarked \%29\#page/n89/mode/2up [access: 25.12.2019]. A colour edition of the facsimile is also available at: http://coptot.manuscriptroom.com/manuscript-workspace [access: 25.12.2019]. 
Similarly, on the following page, on the facsimile marked as $95 \mathrm{M} 568 \mathrm{f.} 47^{\mathrm{r}}$, we come across two Coptic numbers. One of them, written by the right-hand column, i.e. on the outer side, is $\overline{\mathbf{Q}} \mathbf{Z}$. At the left-hand column there is only $\overline{\mathbf{Z}}$, (presumably an "abbreviation" of the numeral $\overline{\mathbf{C}}$ ). Again, here the shape of the letter is slightly different. This may lead to the conclusion that this numeral could have been added later and was not written by the copyist of the manuscript.

\section{Perforations}

The writing material, as it has already indicated in the analyses of the earlier fragments of the manuscript, was not chosen very carefully. The parchment folios often have quite large perforations. On page $90 \mathrm{M} 568 \mathrm{f}$. $44 \mathrm{v}$ (Coptic $\overline{\mathrm{CB}}$ ) one can see a fewcentimetre-wide hole. However, being on the outer side of the folio, it does not affect the Coptic text in any way.

\section{Columns}

The Coptic text was not written in a thoroughgoing manner. The columns have different numbers of verses. The biggest number of lines, 38 , is in the right-hand column on f. $46^{\mathrm{r}}$ (Copt. $\overline{\varphi \epsilon}$ ). The shortest columns, containing 32 lines, are on f. $45^{\mathrm{r}}$ (Copt. $\bar{\varphi} \bar{\Gamma}$, both columns) and f. $45^{\mathrm{v}}$ (Copt. $\overline{4} \bar{\Delta}$, left-hand column).

In our fragment of Deutero-Isaiah, single words were twice written under the columns. The first occurrence is on f. $45^{v}$ (Copt. $\overline{4 \Delta}$ ) under the left-hand column, specifically beneath the letters $\mathrm{N} 2 \omega_{\mathrm{B}}$, that end line 32 . The single word Nim must have completed the expression N2WB NiM ("all things"). The appearance of the word placed under this column can raise a question why a whole verse was not written there since the column actually has 32 lines. The right-hand column is one line longer. Therefore, the scribe could have created a whole line and not a single word to align the length of the columns.

The other single word written below the right-hand column is on f. $46^{\mathrm{v}}$ (Copt.

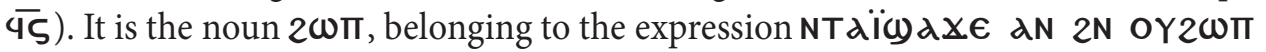
("I have not spoken in secret") and was put precisely under the letters $2 \mathrm{~N} 2 \mathrm{~N}$.

\section{Ornaments}

Manuscript sa 52 contains practically no ornaments other than certain marks that are intended to facilitate the reading of the text. They usually point to these places where a new sentence begins. The devises found in Isa 44:6-45:25 include:

- coronis, sometimes heart-shaped, e.g. on f. $44^{\mathrm{v}}$ (left-hand column), f. $46^{\mathrm{v}}$ (lefthand column) and f. $47^{\mathrm{r}}$ (left-hand column). Its non-typical form occurs on 
the left side of line 16 in the right-hand column on f. $44^{\mathrm{v}}$ (Copt. $\overline{\mathbf{C B}}$ ) in Isa 44:8. It resembles a unicorn's head turned to the left. The sign is written in red ink.

- obelus, appearing on every page,

- pentonkion (five-dot punctuation), also occurring on every page.

A detailed analysis of the "ornaments" shows that some of them were placed on top of the previously written characters. For example, in a few cases on $\mathrm{f} .45^{\mathrm{r}}$, the pentonkion was overwritten with an obelus. In line 9, a coronis was meant to replace the five-dot punctuation. It is difficult to clearly determine whether the "ornaments" come from the copyist of the manuscript or whether they were placed there later. Most of them were written in black and then corrected with red ink.

\section{Letter shapes}

The handwriting in sa 52 is very neat and legible. Some letters are marked in different ways. An example would be T, the horizontal part of which is sometimes extended over the adjacent letters. It can be seen in Isa 44:15 (f. 45 , left-hand column, line 30) in the word $2 \bar{N}$ NOYT $\epsilon$, in Isa 44:17 (f. $45^{\mathrm{r}}$, right-hand column, line 11) in the construction $24 \pi$ व2T4, and in Isa 45:8 (f. 46 , right-hand column, line 20) in the expression $€$ NTA 4 CONTK.

Also, the upper part of the letter $\sigma$ can sometimes be lengthened considerably, for instance in Isa 44:22 (f. 45v, left-hand column, line 15) in the expression NO€ NoY $60 \mathrm{~cm}$, where over Осм there is a horizontal line extended from the letter $\sigma$.

The nomina sacra in the manuscript were not written thoroughly. In Isa 44:6 (f. $44^{v}$, left-hand column, line 31 ), the horizontal line was made not only over the abbreviation $\overline{\mathrm{IH} \lambda}$, but also over the article to create $\overline{\mathrm{M} I \mathrm{IH} \lambda}$. On the other hand, in Isa 44:21 (f. $45^{v}$, left-hand column, line 5) the horizontal line is too short: $\overline{\Pi \mathrm{IH}} \lambda$. It should have been extended over the letter $\lambda$. This can be observed also in Isa 44:23 (the same column, line 19). Isa 44:26 (f. $45^{\mathrm{v}}$, right-hand column, line 17) has the notation $\mathbf{N} \overline{\mathbf{I} \lambda \mathrm{HM}}$, while in Isa 44:28 (the same column, line 30) there is a shortened

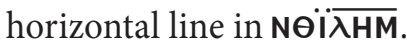

The nomina sacra were not always used as abbreviations. For example, in Isa 44:23 (f. $45^{\mathrm{v}}$, left-hand column, line 24 ), the copyist put the abbreviation $\pi \mathbf{x O} \overline{\mathbf{\epsilon C}}$, whereas in the following verse (the same column, line 27) he wrote the full writing пхо $\mathbf{1}$ ic.

\section{Textual errors}

There are missing letters in some words, as in the examples below:

- In the final part of Isa 44:9 (f. 44v, right-hand column, line 24), we can see C€Naхї $\boldsymbol{\omega} \pi \epsilon$. To correct this expression, we should add the vowel I so as to create

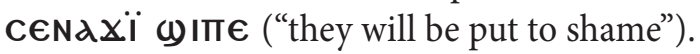


- In Isa 44:20 (f. $45^{\mathrm{r}}$ ), the last word in the right-hand column was written as

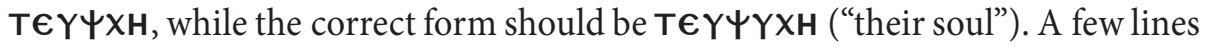
above (line 20), in Isa 44:19, this word occurs in its proper form.

- In Isa 45:8 (f. 46, right-hand column, line 16), we can see the correct wording $\dagger$ oy $\omega$, but further (line 17) - only $\dagger \omega$, which would require the missing vowel oY.

- In Isa 45:8 (f. 46 $6^{\mathrm{r}}$, right-hand column, line 14) there is the incorrect notation of $\mathrm{K} \lambda 00 \lambda$. Its proper form is $\mathrm{K} \lambda \mathrm{\lambda OO} \boldsymbol{\lambda} \epsilon$ ("cloud" ${ }^{\circ}$ ).

- In Isa 45:11 (f. 46v , left-hand column, line 1) in the expression $€$ TP€ாхоєї , the relative conversion $\boldsymbol{\epsilon} \mathbf{T} \boldsymbol{\epsilon} \boldsymbol{\epsilon}$ - was written erroneously: the letter $-\boldsymbol{\epsilon}$ - was

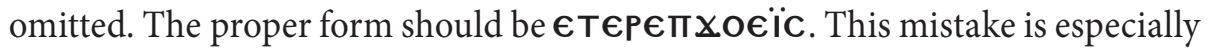
surprising considering that it was made in the first line of a new column of text. There are cases of adding an extra unnecessary letter. Isa 44:26 (f. $45^{\mathrm{v}}$, righthand column, line 13) begins with $€$ TTA20 ЄPAPT $\overline{\mathbf{4}}$, while the right form should be written as $€ T T d 20 \in P d T \overline{4}$. Consequently, the second letter $P$ in the word $€ P-$ APT $\bar{\varphi}$ is not needed.

At several points one can note the tendency to add the letter $\mathbf{N}$, for example, twice in Isa 45:6 (f. 46 , left-hand column, line 37 and right-hand column, line 2) in the same expression $\mathbf{N} \boldsymbol{\epsilon} \mathbf{T} 2 \overline{\mathbf{N}}\{\mathbf{N}\} \mathbf{M M d}^{6}$ as well as in Isa $45: 7$ (f. $46^{\mathrm{r}}$, right-hand col-

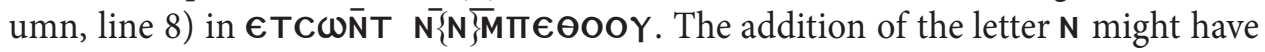
resulted from phonetic reasons. In our edition, all the cases of redundant letters, frequently being the effect of dittography, have been put in braces \{\} .

Moreover, the scribe wrote incorrect letters. Isa 45:14 (f. 46, right-hand column, line 3) contains the expression CENAOY $\omega \omega T$ NAK. Its proper form should be CENAOY $\omega \omega$ T NAK. So, the scribe "did not finish" the letter $\boldsymbol{\omega}$, writing only $\omega$.

In Isa 44:15 (f. 45r, left-hand column, line 27) the author of the manuscript changed the order of two letters in the word NT€P€ 4 PO $2 K \overline{\mathbf{4}}$. A correct version, without a metathesis error, is in the witness of sa 41.16, where we have the reading NTEP€ 4POK $2 \overline{4}$ ("when he burned it").

\section{Final $\mathbf{N}$}

In some cases, the letter $\mathbf{N}$ occurring at the end of a line was written with a horizontal line. ${ }^{7}$ This form can be seen in the following verses:

- At the end of Isa 45:1 (f. 46, left-hand column, line 11), in the expression Na $\mathbf{N}$ $\omega T \bar{M} \bar{d}$. Its obvious correct reading is $N \alpha \omega \omega T \bar{M} d N$.

Crum, Coptic Dictionary, 104a.

The same expression $2 \overline{\mathbf{N}}\{\mathbf{N}\} \bar{M} M \mathbf{d} \mathbf{N} \boldsymbol{N}) \mathbf{d} \mathbf{M}$ MPH written with an additional $\mathbf{N}$ can be seen in Isa 11:11.14 (cf. Isa $1: 11 ; 16: 10$ ).

7 So-called: "Superlinear stroke representing line-final N" (Layton, Coptic Grammar, § 38). 
- In Isa 45:12 (f. 46 written as $2 \ddot{i} 2 \omega$.

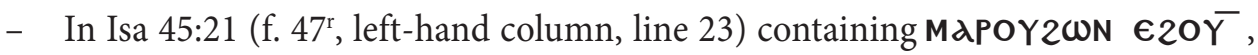
read as MAPOY $2 \omega \mathbf{N} \in 20 Y \mathbf{N}$ ("let them draw near").

In our edition of the manuscript, all the cases of this kind of $\mathbf{N}$ have been put in round brackets: $(\mathrm{N})$.

\section{Corrections in the manuscript}

Fairly numerous verses of manuscript sa 52 contain corrections - frequent additions of missing letters. At the beginning of Isa 44:13 (f. $45^{\mathrm{r}}$, left-hand column, line 11), the letter $\boldsymbol{d}$ in the word or2amwe was added underneath. The handwriting suggests that the addition was made by the author itself.

In Isa 44:17 (f. 45 , right-hand column, line 13), in the expression Md ToYxoï the letter $\mathbf{d}$ was added below. One can also see the traces of a corrected $\mathbf{M}$. In the same verse (line 14), the 2nd pers. sing. pronoun NTOK in the expression Md

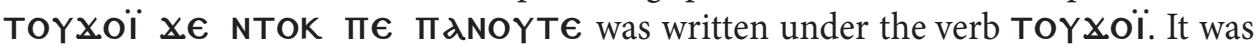
"underlined" with a special sign, which means the author's suggestion to read it directly after the particle $\mathbf{X} \in$. At the beginning of Isa 45:4 (f. 46 , left-hand column, line 26) in the expression $€$ тв $€$ ïak $\omega \mathbf{B}$, the adjacent letters $€$ and $\ddot{i}$ are smaller and probably added as textual corrections.

Right at the beginning of Isa 45:7 (f. $46^{\mathrm{r}}$, right-hand column, line 4), it is difficult to decipher the last letter in the expression $\Pi \boldsymbol{\epsilon} \mathbf{N T A} \mathbf{I C B} T \boldsymbol{C}$ that resembles $\boldsymbol{O}, \boldsymbol{\theta}$ or $\boldsymbol{\epsilon}$. Since here we are dealing with the prenominal form of the verb COBT€ ("be ready," "to prepare") ${ }^{8}$ the final letter must be $\boldsymbol{\epsilon}$. The scribe might have written $\mathbf{O}$ and then changed it into $\boldsymbol{\epsilon}$.

In the expression K€T THYT $\bar{N}$ at the beginning of Isa 45:22 (f. $47^{\mathrm{r}}$, right-hand column, line 1), the letter $Y$ was added above the text.

Summing up, the writing material, unsymmetrical columns, different number of lines, unevenly written nomina sacra as well as errors and corrections testify to a certain negligence in the preparation of manuscript sa 52. On the other hand, the text was written distinctly and has been preserved in good condition to our times, which facilitates its smooth reading.

8 Crum, Coptic Dictionary, 323a. 


\section{List of Manuscripts with the Text of Isa 44:5-45:25 in the Sahidic Dialect of the Coptic Language}

The verses that belonged to the discussed section of the Book of Deutero-Isaiah can be found in the following manuscripts:

Sa 41.15: $17.0 \times 15.5 \mathrm{~cm}$ parchment fragment kept in the National Library in Paris. Its catalogue number is Paris, BN, Copte $131^{6}$ fol. 104. The fragment contains the text of Isa $43: 28^{*}-44: 3 \cdot 5^{*}-6^{*} \cdot 8^{\star}-9^{\star} \cdot 12-13$, which is illegible in a few places. It is dated back to the 9th-10th centuries. ${ }^{9}$ Regrettably, there has been no edition of this fragment. ${ }^{10}$

Sa 41.16: two parchment fragments in one folio catalogued as Paris, BN, Copte $129^{3}$ fol. 155, 156. They belong to the codex containing the text of the Book of Isaiah, the most part of it being destroyed. The whole leaf, which Schüssler identified as sa 41.16, includes the text of Isa 44:13-26. In fragment 156, on its recto side, there are verses $13-15 \mathrm{a}$, followed by $16 \mathrm{~b}-18 \mathrm{a}$. Fragment 155 contains verses $15 \mathrm{~b}-16 \mathrm{a}$ and $18 \mathrm{~b}-20 \mathrm{a}$ on its recto side. On the verso, fragment 156 has verses $20 \mathrm{~b}-21 \mathrm{a}$ and $23 \mathrm{~b}-24 \mathrm{a}$. Fragment 155 on its verso side includes verses $21 \mathrm{~b}-23 \mathrm{a}$ and $24 \mathrm{~b}-26 .{ }^{11}$ Therefore, we can note that fragment 156 shows an earlier text than the one included in manuscript 155. The text was written in two columns, each having 35 lines. In every line there are between 8 and 10 letters. ${ }^{12}$ Its edition has been worked out by Hebbelynck ${ }^{13}$; analysing this edition we can conclude that the text has not been well preserved. Problems with deciphering letters emerge in the right-hand column on its recto side and in the left-hand column on the verso. The fragment of Isa 44:20-21.23-24 can also be found in Maspero's work..$^{14}$ It is dated probably to the 9th century ${ }^{15}$ or the 10th century. ${ }^{16}$ This parchment appears on Vaschalde's list. ${ }^{17}$

Sa 41.17: one parchment leaf sized: ca. 32 x $28 \mathrm{~cm}$; part of the same codex as the previous fragment. ${ }^{18}$ It is housed in the National Library in Vienna under the catalogue number Wien, ÖNB, K 9397. It contains Isa 45:21b-46:13. We focus on Isa $45: 21 \mathrm{~b}-25 \mathrm{a}$. A handwritten edition of manuscript sa 41.17 was prepared by

\footnotetext{
More information in M.E. Porcher, "Analyse des manuscrits," 91.

Schüssler, Sa 21-48, 80.

See Hebbelynck, "Fragments inédits," 191-196.

Detailed information in Hebbelynck, "Fragments inédits," 191 and Schüssler, Sa 21-48, 80-81.

Hebbelynck, "Fragments inédits," 191-196.

Maspero, "Fragments de manuscrits," 223.

Nagel, "Studien zur Textüberlieferung," 148.

Till, "Papyrussammlung," 16 (No. 52).

17 Here we can find detailed information showing the fragmentarity of the preserved text. The verses of Isa 44 are marked as $13 \mathrm{~b}^{*}, 14-16 \mathrm{~b}^{\star}, 17-18,19^{*}, 20 \mathrm{a}^{*}, 20 \mathrm{~b}-23 \mathrm{~b}^{*}$ and $24-26 \mathrm{~b}^{*}$ (Vaschalde, "Ce qui a été publié," [1920] 248).

18 Cf. Schüssler, Sa 21-48, 74-76, 81. Mentions of the manuscript are also given in Till, "Die Coptica," 204, as well as in Till, "Papyrusammlung," 16 (No. 52).
} 
Wessely. ${ }^{19}$ The parchment has also been placed on Vaschalde's ${ }^{20}$ list catalogued as SER $220 .{ }^{21}$ Its comparison with our text of sa 52 shows considerable similarities. Only three differences can be observed in the final verses of Isa 45 .

Sa 197' .2: one leaf parchment sized: $26.6 \times 21.0 \mathrm{~cm}$, written on both sides. This manuscript probably comes from the White Monastery in Sohag, Egypt. ${ }^{22}$ It is dated back to the 9th-11th centuries. ${ }^{23}$ Its recto side contains Rev 22:15-21, while its verso contains Isa 45:16b-20a followed by Jer 38:31-33. ${ }^{24}$ Currently, the manuscript is housed in the British Library in London, under the catalogue number London, BL, Or. 3579 A.31. Vaschalde has marked it as BMC 47, ${ }^{25}$ i.e. in accordance with the number in Crum's catalogue. ${ }^{26}$ The leaf is part of a lectionary that Schüssler identified as sa $197^{\mathrm{L}}$, containing the readings for the Holy Week. ${ }^{27}$ An edition of the Book of Isaiah has been prepared by Schleifer ${ }^{28}$ and Winstedt. ${ }^{29}$ Since Winstedt notes that he had neither enough time to analyse the manuscript in detail nor verified its existing copies, ${ }^{30}$ my work has been based mainly on Schleifer's edition. ${ }^{31}$

P. Mon. Epiph. 26: 19.6 x $9.9 \mathrm{~cm}$ fragment of the ostracon, containing 11 lines. It has two verses of Isa 42:18-19, followed directly by the discussed fragment of Isa 45:24b-25. Unfortunately, the text of these two incomplete verses has been damaged to a significant extent. Only single words can be deciphered. The ostracon was found in the Monastery of Epiphanius at Thebes ${ }^{32}$ and probably comes from the 7th century. ${ }^{33}$ At present, it is housed in the Metropolitan Museum of Art in New York (USA) under the number: 12. 180. 194. On Nagel's list it has been described as MMA 12. 180. 194 (Ostr.): sa ${ }^{\text {exc }} .{ }^{34}$ It has also been considered in the Leuven Database for

\footnotetext{
19 Wessely, Griechische und koptische Texte, No. 220 e-f.

20 Vaschalde, "Ce qui a été publié," [1920] 249.

21 The abbreviation "SER" was given in the Viennese collection containing this manuscript: Sammlung Erzherzog Rainer (cf. Vaschalde, "Ce qui a été publié," [1919] 223).

22 Takla, "The Surviving Remains," 86-87.

23 Cf. Feder, Biblia Sahidica, 45.

24 Winstedt states that it is the recto side that contains the verses of Isa 45:16-20 and Jer 38:31-33 (E.O. Winstedt, "Some Unpublished Sahidic Fragments," 248).

Vaschalde, "Ce qui a été publié," [1920] 249.

Crum, Catalogue, 14 (No. 47).

Schüssler, Sa 185-260, 37.

Schleifer, Sahidische Bibel-Fragmente, 21-23.

Winstedt, "Some Unpublished Sahidic Fragments," 248-249.

Winstedt, "Some Unpublished Sahidic Fragments," 233.

Additional information on sa 197".2 can be found in: Atanassova, "Zu den sahidischen," 615-616; Crum, Catalogue, 14 (No. 47); Feder, Biblia Sahidica, 45 (No. L 31); Schleifer, Sahidische Bibel-Fragmente, 21-22; Schüssler, Sa 185-260, 37-39; Takla, "The Surviving Remains," 86.

32 See Crum -Winlock, The Monastery of Epiphanius.

33 Basis information and photographs of the ostracon are available at: https://www.metmuseum.org/art/ collection/search/170015749 [access: 26.12.2019].

34 Nagel, "Editionen koptischer Bibeltexte," 60.
} 
Ancient Books as LDAB 112534. ${ }^{35}$ However, it has not been catalogued by Schüssler. Its edition has been made by Crum. ${ }^{36}$

In order to better illustrate the contents of the particular manuscripts, the occurrence of the verses from Isa 44:6-45:25 is presented in the table where:

- an " $x$ " means the occurrence of the whole verse,

- an " $(\mathrm{x})$ " means the occurrence of only a fragment of a given verse,

- an empty space in the table means the lack of a given verse in the manuscript.

The contents of the manuscripts are as follows:

Isa 44:6-28

\begin{tabular}{|l|c|c|c|c|c|c|c|c|c|c|c|c|c|c|}
\hline & 6 & 7 & 8 & 9 & 10 & 11 & 12 & 13 & 14 & 15 & 16 & 17 & 18 & 19 \\
\hline Sa 41.16 & & & & & & & & $(\mathrm{x})$ & $\mathrm{x}$ & $\mathrm{x}$ & $(\mathrm{x})$ & $\mathrm{x}$ & $\mathrm{x}$ & $(\mathrm{x})$ \\
\hline
\end{tabular}

\begin{tabular}{|l|c|c|c|c|c|c|c|c|c|}
\hline & 20 & 21 & 22 & 23 & 24 & 25 & 26 & 27 & 28 \\
\hline Sa 41.16 & $(\mathrm{x})$ & $\mathrm{x}$ & $\mathrm{X}$ & $(\mathrm{x})$ & $\mathrm{x}$ & $(\mathrm{x})$ & $(\mathrm{x})$ & & \\
\hline
\end{tabular}

Isa 45

\begin{tabular}{|l|l|l|l|l|l|l|l|l|l|l|l|l|l|l|l|}
\hline & 1 & 2 & 3 & 4 & 5 & 6 & 7 & 8 & 9 & 10 & 11 & 12 & 13 & 14 & 15 \\
\hline Sa 41.17 & & & & & & & & & & & & & & & \\
\hline Sa $197^{\mathrm{L}} .2$ & & & & & & & & & & & & & & & \\
\hline P. Mon. Epiph. 26 & & & & & & & & & & & & & & & \\
\hline
\end{tabular}

\begin{tabular}{|l|c|c|c|c|c|c|c|c|c|c|}
\hline & 16 & 17 & 18 & 19 & 20 & 21 & 22 & 23 & 24 & 25 \\
\hline Sa 41.17 & & & & & & $(\mathrm{x})$ & $\mathrm{x}$ & $\mathrm{x}$ & $\mathrm{x}$ & $(\mathrm{x})$ \\
\hline Sa 197.2 & $(\mathrm{x})$ & $\mathrm{x}$ & $(\mathrm{x})$ & $(\mathrm{x})$ & & & & & & \\
\hline P. Mon. Epiph. 26 & & & & & & & & & $(\mathrm{x})$ & $(\mathrm{x})$ \\
\hline
\end{tabular}

The tables show that outside manuscript 52, approximately half the text of Isa 44:6-45:25 can be found in other manuscripts. In addition, a large part of the text is fragmentary. Out of the 48 verses analysed in this study, only 11, i.e. less than $25 \%$, occur as complete outside manuscript sa 52 . Thus, it seems that these juxtapositions support the purposefulness of the proposed study.

35 See https://www.trismegistos.org/text/112534 [access: 26.12.2019]. See also https://atlas.paths-erc. eu/manuscripts/1600 [access: 26.12.2019].

Crum - Evelyn White, The Monastery of Epiphanius, 6 (No. 26). 


\section{The Sahidic text of Isa 44:6-45:25}

As in the case of the previous chapters the following signs have been introduced in the edition of the Coptic text:

$<$ pointed brackets indicating that the text has been completed so that it can be properly understood,

\{\} braces indicate the scribe's redundant letters (frequently being the effect of dittography),

$>\quad$ sign indicating the lack of the given form in the manuscript whose number is given beside it, exclamation mark in superscript suggests a more correct reading,

(N) shows the places in which the letter $\mathbf{N}$, occurring at the end of the line, was signalised by a stroke,

I/ sign indicating a letter added subsequently by the scribe above the line,

$/$ sign indicating a letter added subsequently by the scribe below the line.

The text of Isa 44:6-45:25 in the Sahidic dialect of the Coptic language reads as follows:

Isaiah 44:6-28

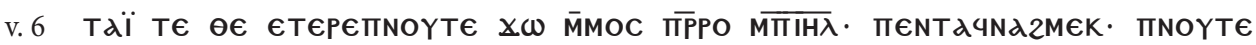

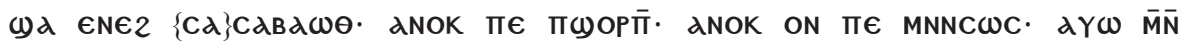

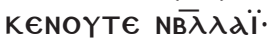

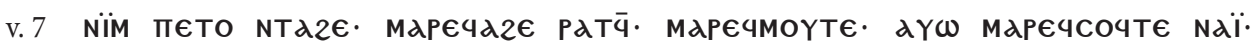

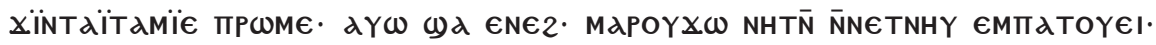

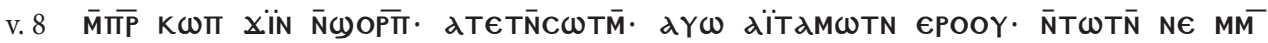

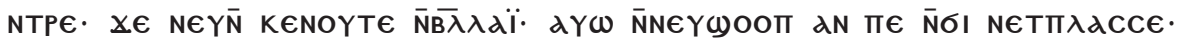
aYW NETMOXT.

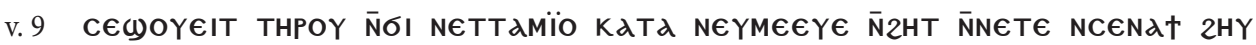

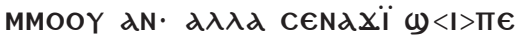

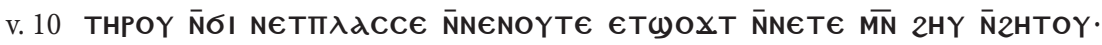

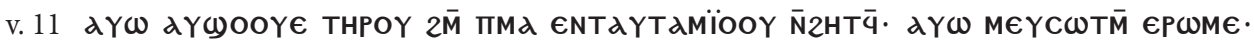

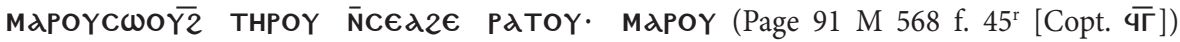

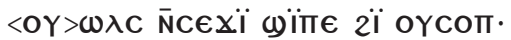

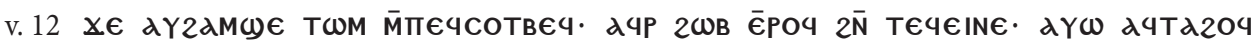

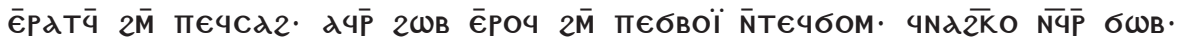

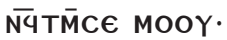

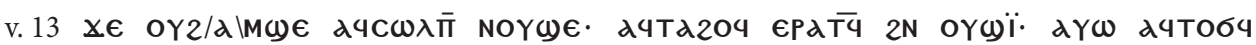

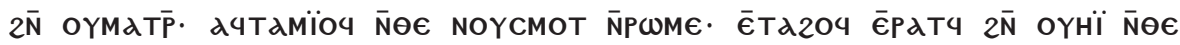
$\bar{M} \Pi$ त् NOYPCME .

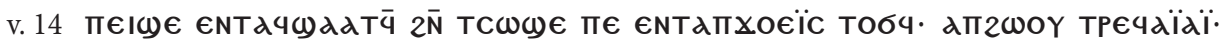

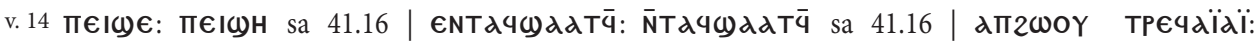

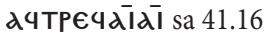




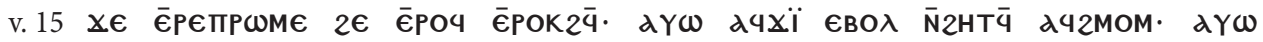

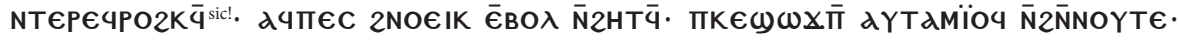
dYOYWWT NAY.

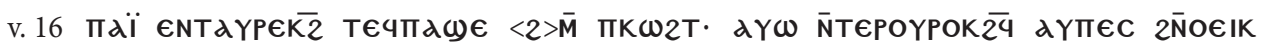

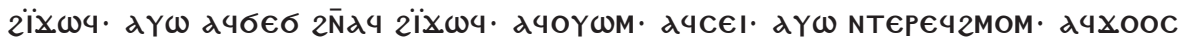

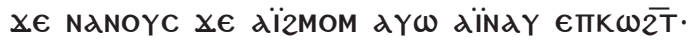

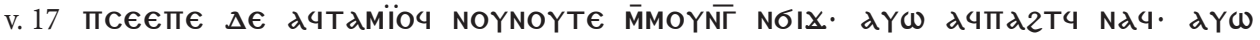

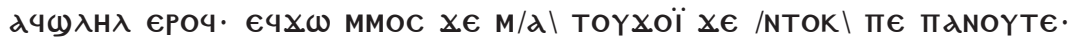

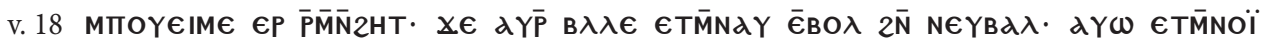
$2 \overline{\mathrm{M}} \Pi \epsilon$ п्HT.

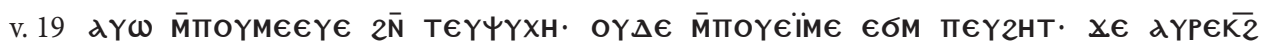

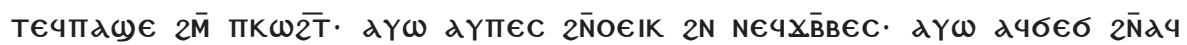

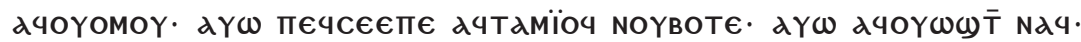

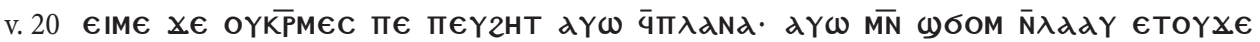

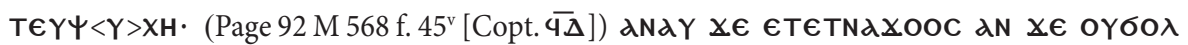

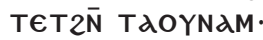

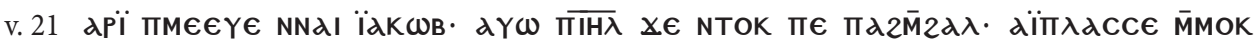

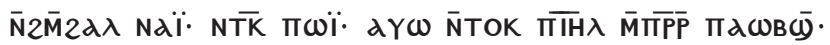

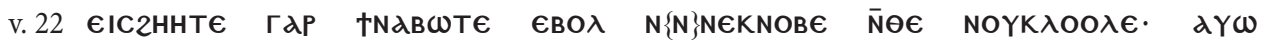

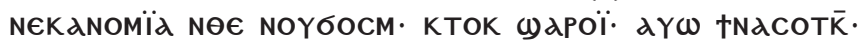

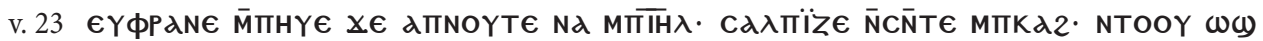

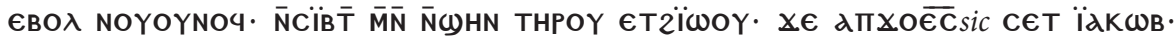

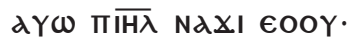

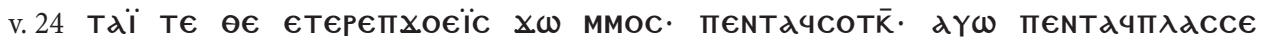

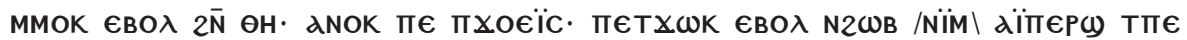
€BO

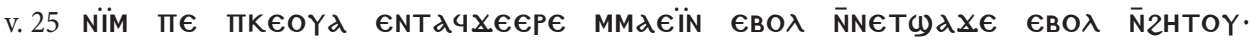

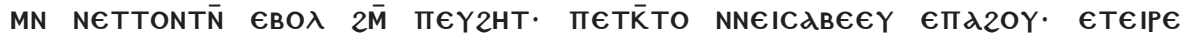

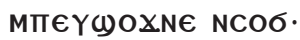

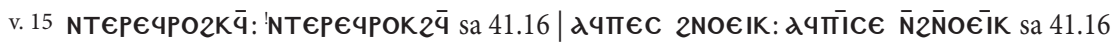

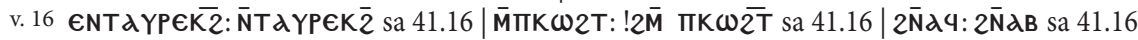

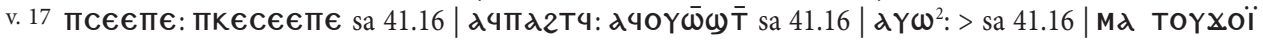
$x \in:>$ sa 41.16

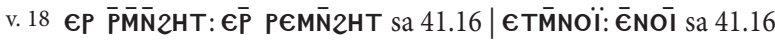

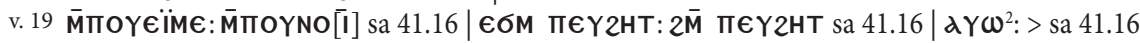

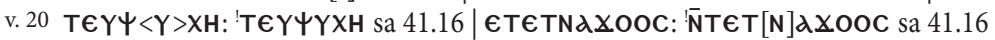

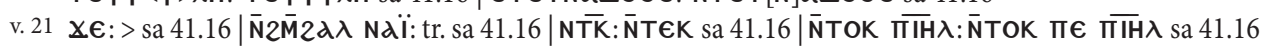

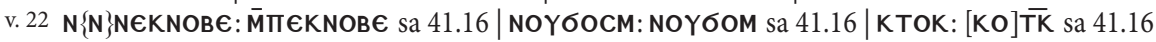

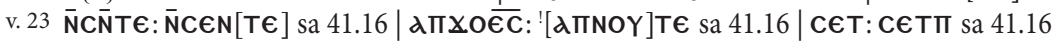

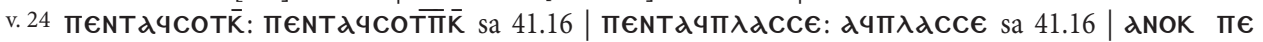

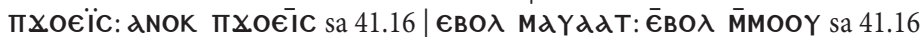

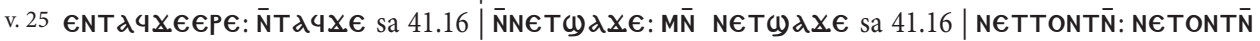

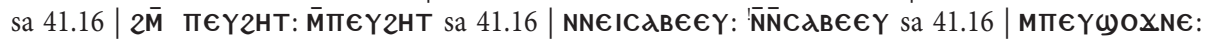
$\bar{N} N \in Y \omega O X N €$ sa 41.16 


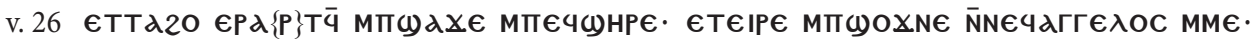

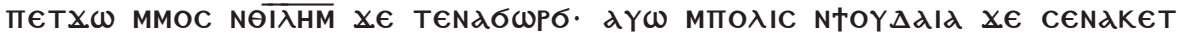

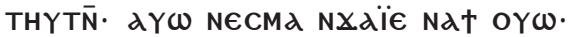

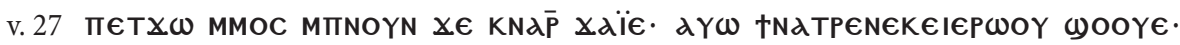

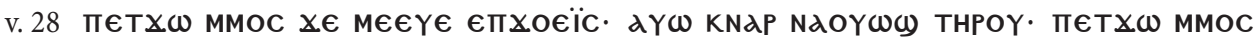

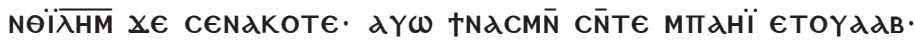

Isaiah 45:1-25

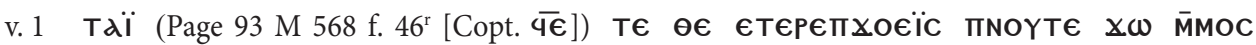

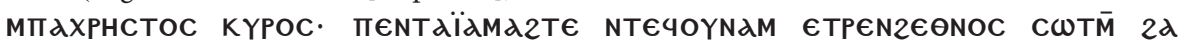

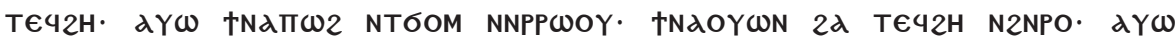

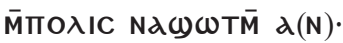

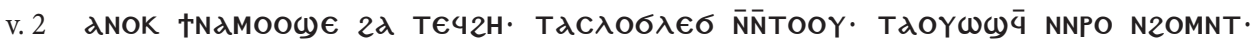

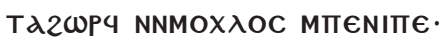

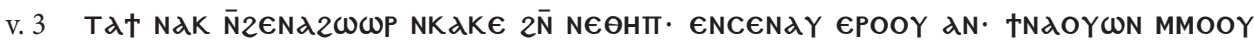

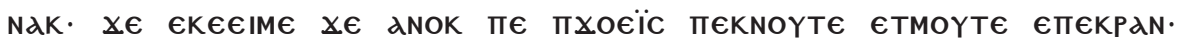

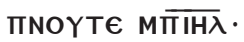

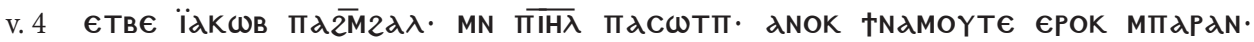

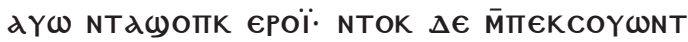

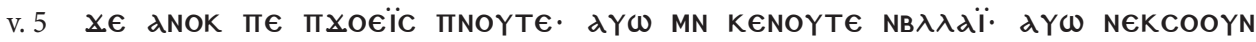
MMOII dN $\Pi \epsilon$.

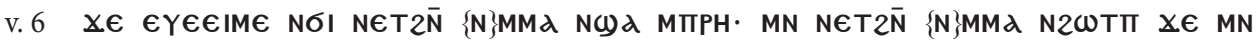
$\sigma \in \mathrm{NB} \lambda \lambda \lambda \boldsymbol{\alpha}$.

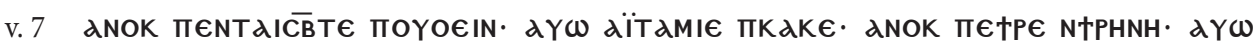

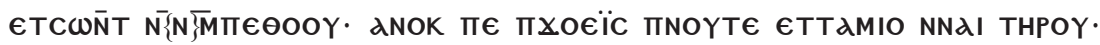

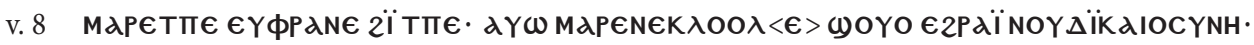

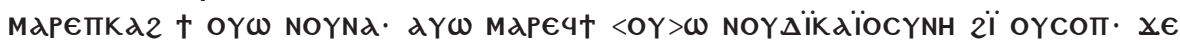

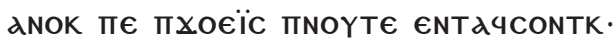

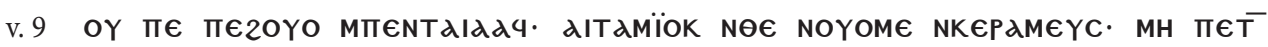

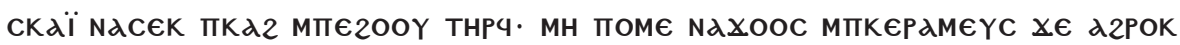

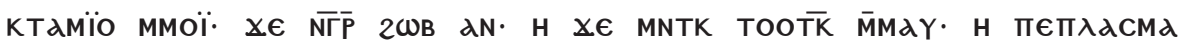

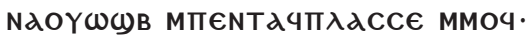

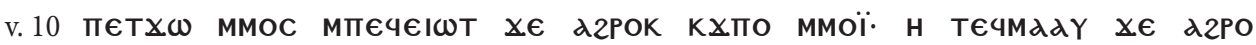
T€† NdגK€.

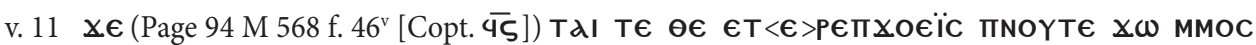

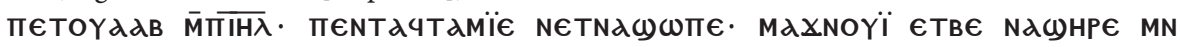

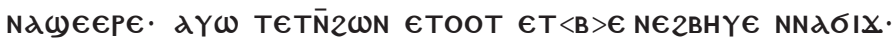

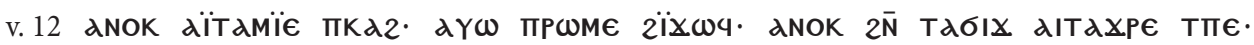

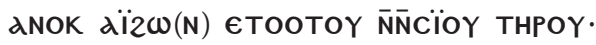

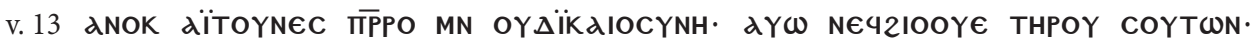

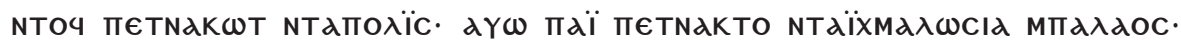

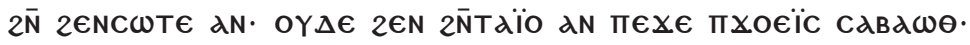

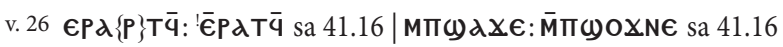




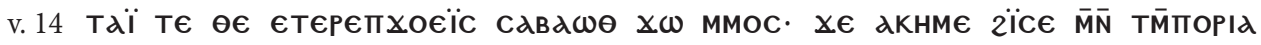

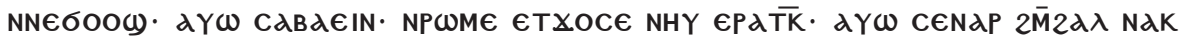

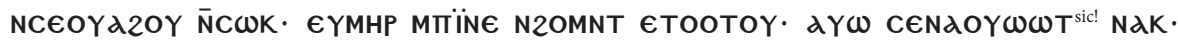

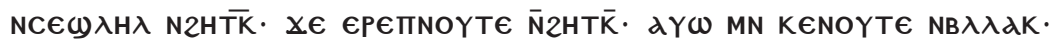

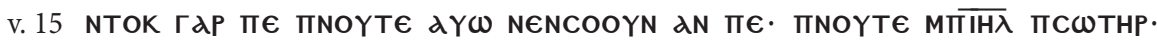

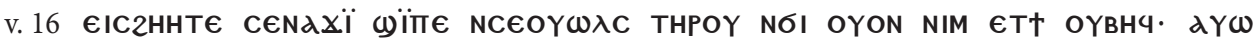

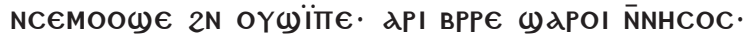

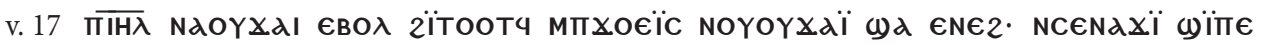

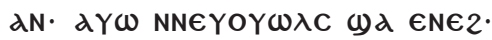

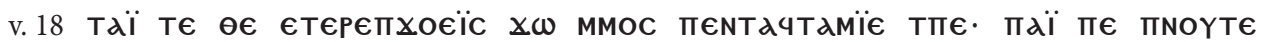

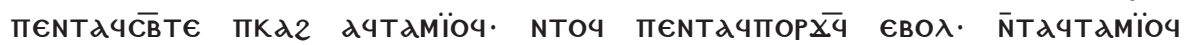
dN $€$ IXINXH· $2 \lambda \lambda \lambda$ ЄTPEYOYW2 N2HTY. $\mathrm{NB} \lambda \lambda \alpha \ddot{i} \cdot$

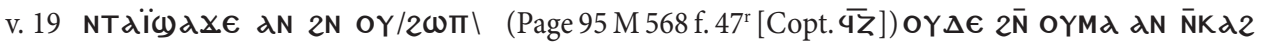

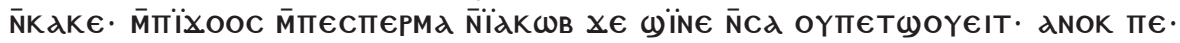

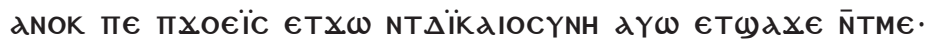

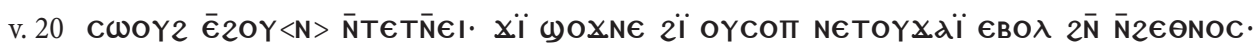

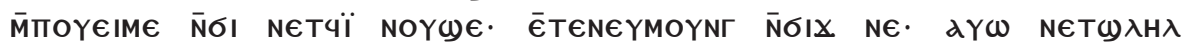
EZENNOYTE ETE NCENATANZOOY AN.

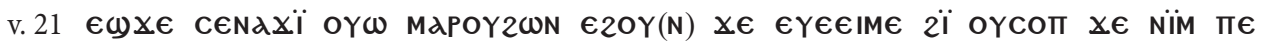

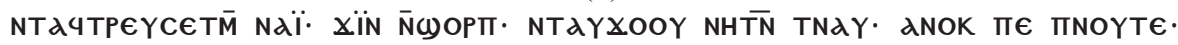

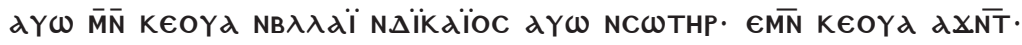

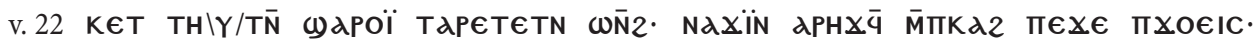

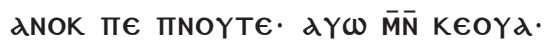

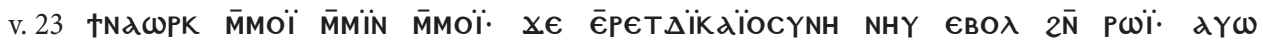

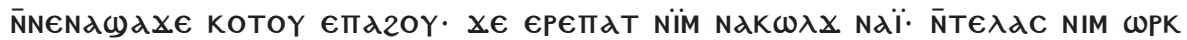
MTINOYTE

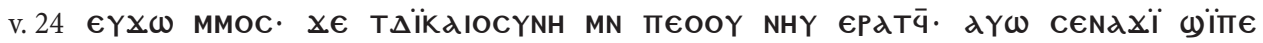
THPOY. NOI NETTWPX MMOOY

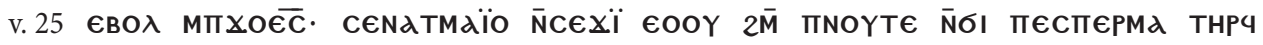
$\bar{N} N \in \omega H P € \overline{M \pi I H \lambda}$.

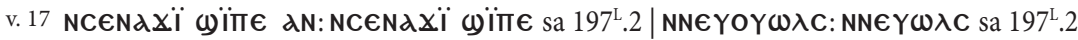

v. 18 NTO4 п€NT дNOK $\pi €:[$ גNOK $\Pi] \epsilon$ пNOYT€ sa $197^{\mathrm{L}} .2$

v. 19 M

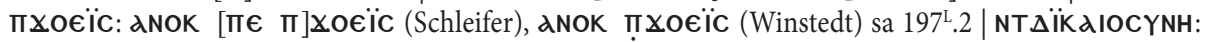
$\mathrm{N}[\mathrm{OY}] \Delta \ddot{\mathrm{IiK}} \mathrm{AIOCYNH}$ sa $197^{\mathrm{L}} \cdot 2$

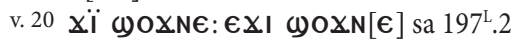

v. $21 \bar{M} \bar{N}$ KEOYd:MN K[ENOYT€] sa 41.17

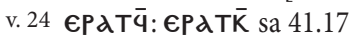

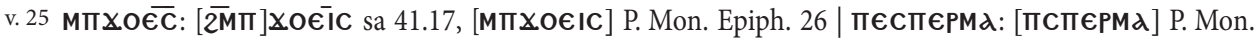

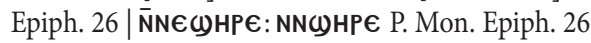




\section{An English Translation of the Sahidic Text}

In translating the Coptic text of Isa 44:6-45:25 into English I have used NETS. ${ }^{37}$ The text of the Septuagint, being the basis of my philological investigations, has been taken from the critical edition by Ziegler. ${ }^{38}$

The English translation of Isa 44:6-45:25 from the Sahidic dialect of the Coptic language is as follows:

\section{Chapter 44}

v. 6 Thus says God, the king of Israel, who delivered you, ${ }^{39}$ eternal $^{40}$ God Sabaoth: I am first, and ${ }^{41} \mathrm{I}$ am also ${ }^{42}$ after these things; and $d^{43}$ besides me there is no other ${ }^{44}$ god.

v. 7 Who is like me? Let him stand; let him call, and let him make ready for me, since ${ }^{45}$ I have made man $a n d^{46}$ forever, and ${ }^{47}$ let them declare to you the things that are coming before they come. ${ }^{48}$

v. 8 Do not cover yourselves from the beginning; you gave ear and I declared it to you. ${ }^{49}$ You are witnesses whether there $w^{50}{ }^{50}$ god besides me, and those who fashion and carve were not..$^{51}$

v. 9 All who do the things following ${ }^{52}$ their minds, ${ }^{53}$ are vain.$^{54}$ The things ${ }^{55}$ will not profit them. But they all ${ }^{56}$ will be put to shame,

37 The motives of using NETS as well as other introductory remarks to the English translation are the same as in the analysed text of Isa 40 (see Bąk, Isa 40, 84).

38 Ziegler, Septuaginta.

39 NETS: him $\rightarrow$ T 7

40 Om. in NETS $\rightarrow$ T 1

41 Lit. om. in sa $52 \rightarrow \mathrm{T} 2$

42 Lit. om. in NETS $\rightarrow \mathrm{T} 1$

43 Om. in NETS $\rightarrow$ T 1

44 Om. in NETS $\rightarrow$ T $1, \mathrm{~T} 6$

45 NETS: inasmuch as (LXX: ’à' oũ).

46 Om. in NETS $\rightarrow$ T 1

47 Lit. om. in sa $52 \rightarrow \mathrm{T} 2$

48 Tr. $\rightarrow$ T 6

49 NETS: Do not cover yourselves; did you not give ear from the beginning, and I declared it to you? (see the commentary on the verse).

50 NETS: is $\rightarrow \mathrm{T} 7$

51 NETS: and they were not formerly. 9. All who fashion and carve are vain. The proposed version, as a rendering from Coptic, can also be found as a possible translation in the footnote in NETS: and those who fashion and carve were not formerly. In Coptic om. formerly $\rightarrow \mathrm{T} 2$

52 NETS: that are in $\rightarrow \mathrm{T} 1$

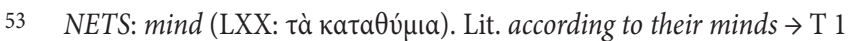

54 Om. in NETS, which results from a different division of verses.

55 Lit. which (= NETS)

56 Om. in NETS, which results from a different division of verses (see the commentary on the verse 10). 
v. 10 who fashion $\operatorname{gods} s^{57}$ or $^{58}$ cast useless things,

v. 11 and all have withered where they made them, ${ }^{59}$ and they cannot hear people. ${ }^{60}$ Let them all assemble and stand together; let them be disgraced and put to shame together.

v. 12 Because $a n^{61}$ artisan sharpened $h i s^{62}$ iron, he fashioned it with $h i s^{63} \mathrm{ax}^{64}$ and bored $\mathrm{it}^{65}$ with a gimlet, he fashioned it with his strong arm; he also ${ }^{66}$ will become hungry and weak and will not drink water.

v. 13 Having cut $^{67}$ a wood, ${ }^{68}$ the artisan set it up with a measure and arranged it with glue; he made it like the form of a man, to set it up in a house like human beauty ${ }^{6}$

v. 14 This wood which he cut in the country is that one that the Lord planted ${ }^{70}$ and the rain made $i t^{71}$ grow,

v. 15 so that man could find it to burn it. ${ }^{72}$ And taking part of it, he warmed himself, and when he burned ${ }^{73} i{ }^{74} e^{75}$ baked breads ${ }^{76}$ on it. $^{77}$ But the rest they fashioned into gods, and they do obeisance to them.

v. 16 Half of it they burned $u p^{78}$ in the fire, and when they were burning it [= wood], they baked breads on it, ${ }^{79}$ and he roasted ${ }^{80}$ meats ${ }^{81}$ over it, he ate it and was satisfied. And having warmed himself, he said, "I am pleased, for I have been warmed and have seen the fire!"

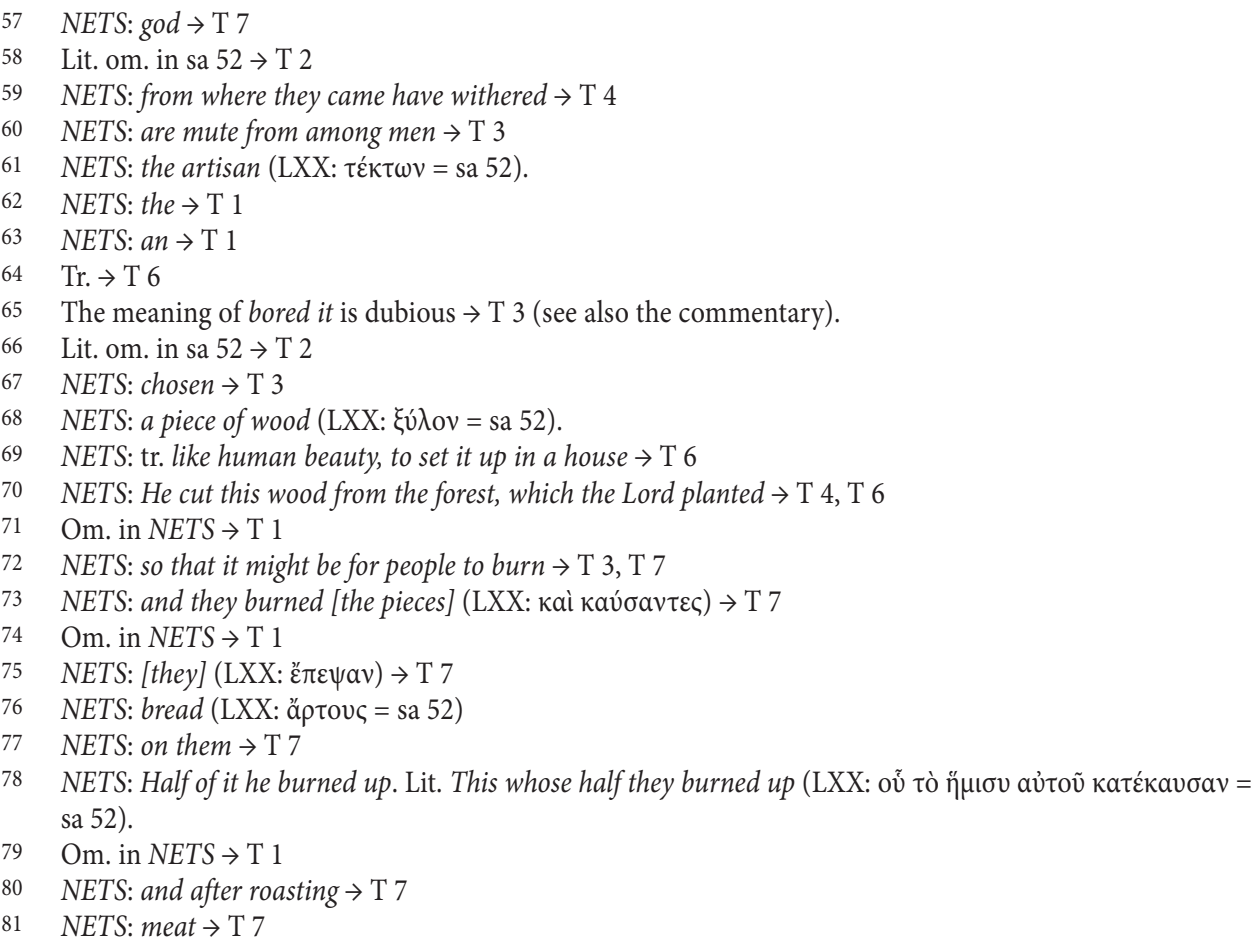


v. 17 The rest he made into a graven god and did $^{82}$ obeisance to it, and he prayed ${ }^{83}$ to it, ${ }^{84}$ saying, "Rescue me, for you are my god!" 85

v. 18 They did not know how to think, because they were blinded so as not to see with their eyes and understand with their heart.

v. 19 And they have $e^{86}$ not considered ${ }^{87}$ in their ${ }^{88}$ soul nor known ${ }^{89}$ in their mind $^{90}$ that half of it the $y^{91}$ burned in the fire and that they $y^{92}$ baked breads $s^{93}$ on $^{94}$ its coals and $h e^{95}$ roasted meats ${ }^{96}$ and ate them $^{97}$ and that the rest of it he made into an abomination, and he did ${ }^{98}$ obeisance to it.

v. 20 Know that their heart is ashes, and he is going astray, ${ }^{99}$ and no one is able to deliver their ${ }^{100}$ soul. See, that you will not say, ${ }^{101}$ "There is a lie in my right hand".

v. 21 Remember these things, O Iakob and Israel, for you are my servant; I formed you as my servant, you are mine, ${ }^{102}$ and you, Israel, do not forget me.

v. 22 For see, I have blotted out your sins ${ }^{103}$ like a cloud and your acts of lawlessness ${ }^{104}$ like darkness; return to me, and I will redeem you.

v. 23 Rejoice, $\mathrm{O}$ heavens, because God has had mercy on Israel; trumpet, O foundations ${ }^{105}$ of the earth; shout for joy, O mountains ${ }^{106}$, the hills and all the trees that are in ${ }^{107}$ them, because the Lord ${ }^{108}$ has redeemed Iakob, and Israel will be glorified!

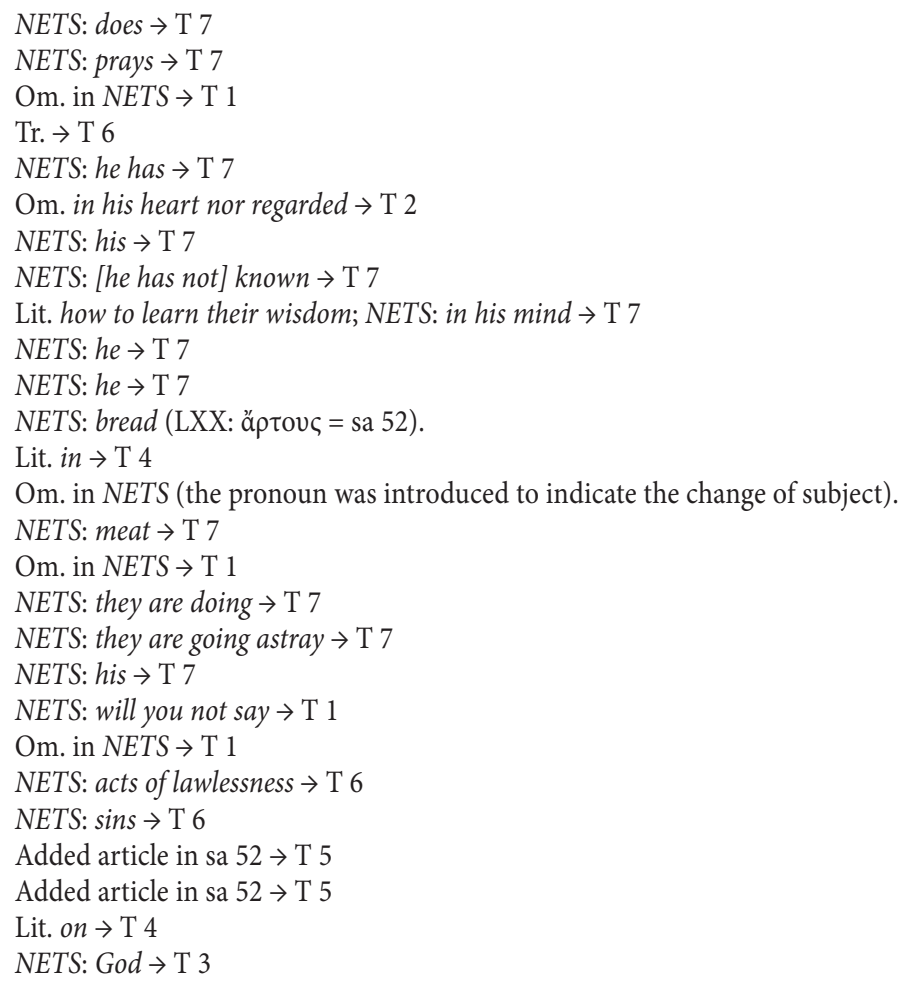


v. 24 Thus says the Lord, who redeems you, who forms you from the womb: I am ${ }^{109}$ the Lord, who accomplishes all things; I alone stretched out heaven, and I bolstered the earth.

v. 25 Who else scattered ${ }^{110}$ the signs given by ${ }^{111}$ ventriloquists and the divinations from their ${ }^{112}$ heart, turning the wise ${ }^{113}$ backward and ${ }^{114}$ making their counsel foolish

v. 26 and confirming the word $^{115}$ of his servant and proving true the counsel of his messengers? The one who says to Ierousalem, "You shall be inhabited," and to the cities of Judea, "You shall be built," and her deserted places shall bring forth,

v. 27 who says to the deep, "You will become desolate, and I will dry up your rivers,"

v. 28 who tells the lord ${ }^{116}$ to be wise and [says], "You ${ }^{117}$ shall carry out all my wishes," who says to Ierousalem, "You shall be built, and I will lay the foundations of my holy house."

\section{Chapter 45}

v. 1 Thus says the Lord God to my anointed, Cyrus, whose right hand I have grasped so that nations will obey ${ }^{118}$ before him, and I will break through the strength of kings; I will open doors before him - and cities shall not be closed:

v. 2 I will go before him ${ }^{119}$ and level mountains; I will break in pieces doors of bronze and break off bars of iron,

v. 3 and ${ }^{120}$ I will give you dark treasures from what is secret, unseen. I will open ${ }^{121}$ them $^{122}$ for you so that you may know that I am the Lord, your ${ }^{123}$ God, who calls your name, the God of Israel. ${ }^{124}$

v. 4 For the sake of Iakob, my servant, ${ }^{125}$ and Israel my chosen, I will call you by my name and receive you, but you did not know me,

\footnotetext{
109 Lit. om. in LXX $\rightarrow$ T 1

110 NETS: will scatter $\rightarrow \mathrm{T} 7$

111 Lit. the signs from $\rightarrow \mathrm{T} 4$

112 NETS: the $\rightarrow$ T 1

113 Lit. these wise ones $\rightarrow$ T 1

114 Lit. om. in sa $52 \rightarrow \mathrm{T} 2$

115 NETS: words $\rightarrow$ T 7

116 NETS: Cyrus $\rightarrow$ T 3

117 NETS: $\mathrm{He} \rightarrow \mathrm{T} 7$

118 Lit. will hear $\rightarrow \mathrm{T} 3$

119 NETS: $y$ ou $\rightarrow \mathrm{T} 7$

120 Lit. om. in sa $52 \rightarrow$ T 2

121 NETS: hidden, unseen ones I will open. Different division of verses (see the commentary).

122 Om. in NETS $\rightarrow \mathrm{T} 1$

123 Om. in NETS $\rightarrow$ T 1

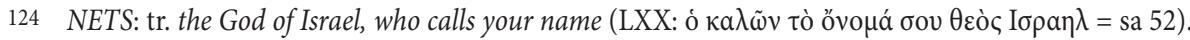

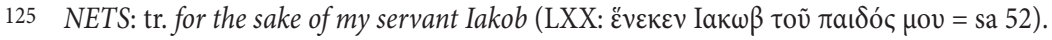


v. 5 because I am the Lord God, and there is no other god besides me, and you did not know me,

v. 6 so that they who are from the rising of the sun and from its going down may know that there is no other ${ }^{126}$ besides $\mathrm{me}^{127}$;

v. 7 I am the one who has prepared light and $I^{128}$ made darkness. I am the one ${ }^{129}$ who makes peace and creates evils; I am the Lord $G o d^{130}$ who does all these things.

v. 8 Let heaven rejoice from above, and let the clouds shower down righteousness; let the earth bring forth mercy, and let it bring forth righteousness as well; I am the Lord God ${ }^{131}$ who created you.

v. 9 What better thing have I made? ${ }^{132}$ I have formed you ${ }^{133}$ like potter's clay. Shall the plowman plow the earth all day ${ }^{134}$ ? Shall the clay say to the potter, "What are you doing, since you are not working, nor do you have your ${ }^{135}$ hand ${ }^{136 " ? ~ O r ~ s h a l l ~}$ the formed thing reply to the one who has formed it? ${ }^{137}$

v. 10 [It is like] the one who says to his ${ }^{138}$ father, "What $d o^{139}$ you beget?" or ${ }^{140}$ to his ${ }^{141}$ mother, "With what are you in labor?"

v. 11 Because thus says the Lord God, the Holy One of ${ }^{142}$ Israel ${ }^{143}$, the one who has made the things that are coming: Ask me about my sons and about ${ }^{144}$ my daughters, and command me concerning the works of my hands.

v. 12 I made the ${ }^{145}$ earth and humankind upon it; I bolstered heaven with my hand; I commanded all the stars.

v. 13 I have raised the king ${ }^{146}$ with righteousness, and all his paths shall be straight; he shall build my city and turn back the captivity of my people, not with ransom or with gifts, said the Lord Sabaoth.

\footnotetext{
126 NETS: no one $\rightarrow \mathrm{T} 1$

127 Om. I amm the Lord God, and there is no other $\rightarrow \mathrm{T} 2$

128 Om. in NETS $\rightarrow \mathrm{T} 7$

129 Om. in NETS $\rightarrow$ T 1

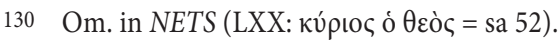

131 Om. in NETS $\rightarrow \mathrm{T} 1$

132 Om. in NETS: $\rightarrow \mathrm{T} 1$

133 NETS: formed $\rightarrow \mathrm{T} 1$

134 Om. in NETS: $\rightarrow$ T 1

135 Om. in NETS $\rightarrow$ T 1

136 NETS: hands $\rightarrow \mathrm{T} 7$

137 Om. in NETS $\rightarrow$ T 1

138 Om. in LXX $\rightarrow$ T 1

139 NETS: will $\rightarrow \mathrm{T} 7$

140 NETS: and $\rightarrow \mathrm{T} 3$

141 Om. in LXX $\rightarrow$ T 1

142 Lit. om. in $\mathrm{LXX} \rightarrow \mathrm{T} 7$

143 Tr. $\rightarrow$ T 6

144 Lit. om in sa $52 \rightarrow \mathrm{T} 2$

145 Om. in LXX $\rightarrow$ T 5

146 NETS: him $\rightarrow$ T 3
} 
v. 14 Thus says the Lord Sabaoth: Egypt has worked hard, as has the commerce of the Ethiopians. And the lofty men of Seboin ${ }^{147}$ shall come over to you, and they shall be your slaves; they shall follow behind you bound in copper ${ }^{148}$ handcuffs. They will do obeisance to you and pray in you, because God is in you, and ${ }^{149}$ there is no other ${ }^{150}$ god besides you.

v. 15 For you are God, and we did not know it, O God of Israel, Savior. ${ }^{151}$

v. 16 For see, ${ }^{152}$ all who oppose him shall be ashamed and disgraced, and they shall go in shame. Dedicate yourselves ${ }^{153}$ to me, you islands!

v. 17 Israel will be $e^{154}$ saved by the Lord with everlasting salvation; they shall not be ashamed or ${ }^{155}$ disgraced forever.

v. 18 Thus says the Lord, who made heaven - this is the God who set in order ${ }^{156}$ the earth and ${ }^{157}$ made it; he himself marked its limits; he did not make it to be empty but to be inhabited: I am, I am, ${ }^{158}$ and there is no other besides $m e^{159}$.

v. 19 I have not spoken in secret nor in a dark place of the earth; I did not say to the offspring of Iakob, "Seek a vain thing." I am, I am the ${ }^{160}$ Lord, speaking righteousness and declaring truth.

v. 20 Assemble yourselves, and come, take counsel together, you ${ }^{161}$ who are being saved from among the nations! They did not know - those who lift up the wood, namely their hand-made things ${ }^{162}$ and pray ${ }^{163}$ to gods that will ${ }^{164}$ not save ${ }^{165}$ them. ${ }^{166}$

v. 21 If they will declare it, let them draw near so that they may know together who made from the beginning these things that are to be heard. When ${ }^{167}$ it was declared

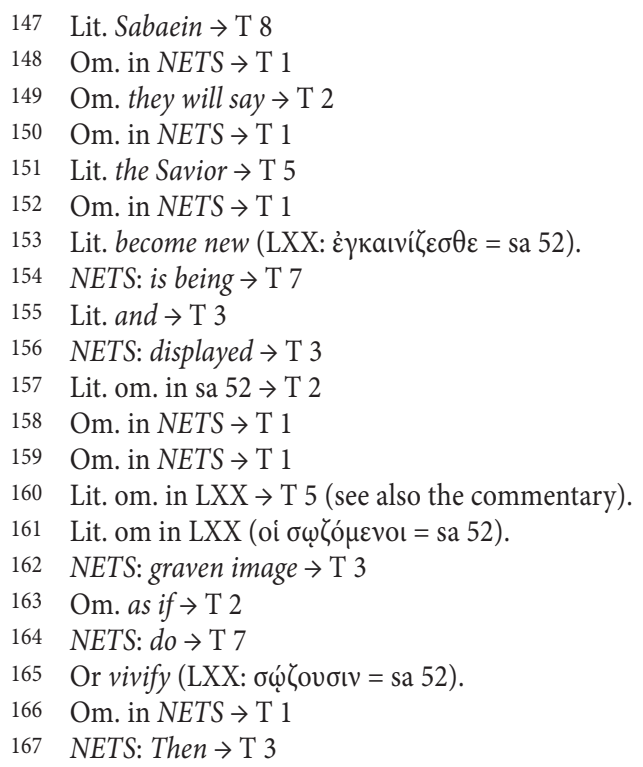


to you, I am God, and there is no other righteous one and savior ${ }^{168}$ besides me? There is no other ${ }^{169}$ except me.

v. 22 Turn to me, and you shall live, ${ }^{170}$ you who are from the end of the earth! - says the Lord. ${ }^{171} \mathrm{I}$ am God, and there is no other.

v. 23 By myself I swear, "Righteousness ${ }^{172}$ shall go forth from my mouth; and ${ }^{173}$ my words shall not be turned back, because to me every knee shall bow and every tongue shall swear to God, ${ }^{174}$

v. 24 saying, Righteousness and glory shall come to him, and all who separate themselves shall be ashamed."

v. 25 By the Lord shall they be justified, and all the offspring of the sons of Israel shall be glorified in God.

\section{Tables of Language Differences}

The differences between the text of the Septuagint and its Coptic translation will be presented in the following order: additions (Table 1), omissions (Table 2) found in the Coptic text, the use of different vocabulary (Table 3), changes of prepositions (Table 4) and articles (Table 5), ${ }^{175}$ changes in word order (Table 6) ${ }^{176}$ and semantic changes (Table 7). ${ }^{177}$ The last table shows the Greek borrowings appearing in the Coptic text of Isa 41 (Table 8). ${ }^{178}$

\footnotetext{
168 Om. in NETS (see the commentary).

169 NETS: righteous one or savior (see the same commentary) $\rightarrow \mathrm{T} 1$

170 NETS: you shall be saved $\rightarrow$ T 3

171 Om. in NETS $\rightarrow$ T 1

172 NETS: Verily righteousness $\rightarrow \mathrm{T} 2$

173 Om. in NETS $\rightarrow$ T 1

174 NETS: acknowledge God $\rightarrow$ T 3 (see also the commentary).

175 Omitting or adding an article does not necessarily result from the translator's intention to interfere in the content. The semantic rules frequently (especially in Coptic) decide about the omission of an article. Therefore, it would be no "material" sense to list all the places where the Coptic translation is not faithful to all the articles occurring in the Greek LXX. Table 5 only shows selected examples.

176 The differences in word order do not always have to reflect the real changes introduced by the Coptic translator. They can often depend on the syntactic rules according to which, e.g. the direct object usually appears immediately after the verb (see Isa 41:18.19) (cf. Layton, Coptic Grammar, \$ 182).

177 Here we have included the grammatical and semantic changes (e.g. number, tense, person, gender, etc.).

178 For remarks concerning the tables see Bąk, Isa 41, 76.
} 
Table 1. Additions in the Coptic text

\begin{tabular}{|c|c|c|}
\hline $44: 6$ & $\theta \varepsilon o ̀ \varsigma ~ \sigma \alpha \beta \alpha \omega \theta:$ God Sabaoth & 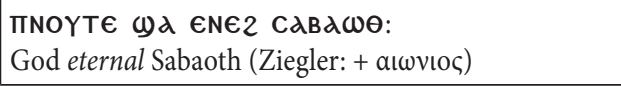 \\
\hline $44: 6$ & $\dot{\varepsilon} \gamma \grave{\omega} \mu \varepsilon \tau \grave{\alpha} \tau \alpha \tilde{\tau} \tau \alpha:$ I am after these things & $\begin{array}{l}\text { ANOK ON } \Pi \epsilon \text { MNNCWC: } \\
\text { I am also after these things (> Ziegler) }\end{array}$ \\
\hline $44: 6$ & 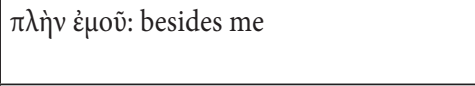 & $\begin{array}{l}\text { pr. } \lambda \gamma \omega \text { (Ziegler: pr. kal without any references } \\
\text { to Coptic) }\end{array}$ \\
\hline $44: 6$ & 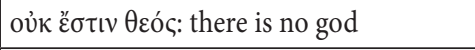 & $\overline{\mathbf{M}} \overline{\mathrm{N}}$ K€NOYT€: there is no other god (> Ziegler) \\
\hline $44: 7$ & 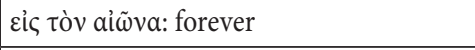 & 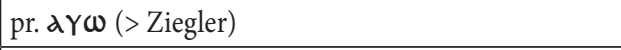 \\
\hline 44:9 & 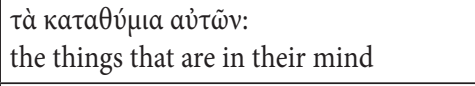 & $\begin{array}{l}\text { KдTd N€YM€€Y€ } \overline{\mathbf{N}} 2 \mathrm{HT} \text { : } \\
\text { lit. according to their minds (Ziegler: pr. кaTa Co) }\end{array}$ \\
\hline 44:12 & 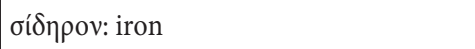 & м̈пєчсотвєч: his iron (> Ziegler) \\
\hline $44: 12$ & 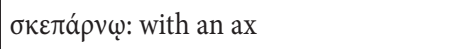 & $2 \overline{\mathbf{N}}$ T€ч€IN€: with his ax (> Ziegler) \\
\hline 44:14 & 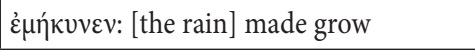 & TP€४גї̈I: [the rain] made it grow (> Ziegler) \\
\hline 44:15 & 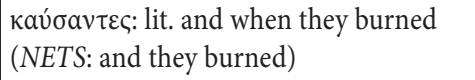 & NT€Р€чРОК $2 \overline{\mathbf{4}}:$ when he burned it (> Ziegler) \\
\hline 44:16 & $\dot{\varepsilon} v \pi v \rho i$ : in the fire & 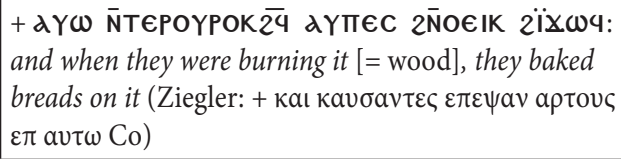 \\
\hline 44:17 & 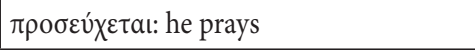 & 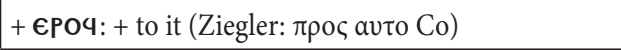 \\
\hline $44: 19$ & 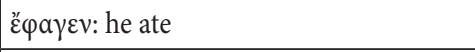 & дчоүомоү: he ate them (= meats) (> Ziegler) \\
\hline 44:19 & $\pi \rho \circ \sigma \kappa v v o v ̃ \sigma t v:$ they are doing obeisance & 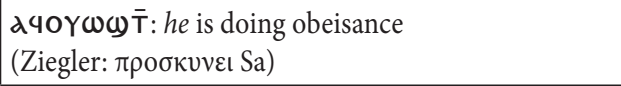 \\
\hline $44: 20$ & 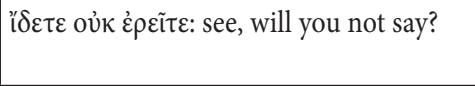 & 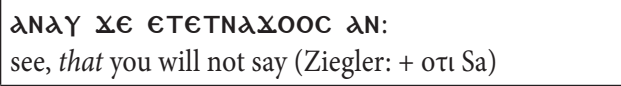 \\
\hline $44: 21$ & 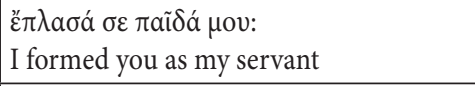 & 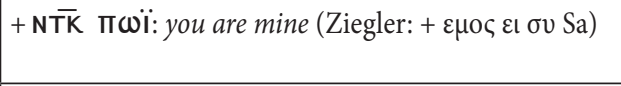 \\
\hline $44: 24$ & $\dot{\varepsilon} \gamma \omega \grave{\omega}$ kúpıoc: I [am] the Lord & 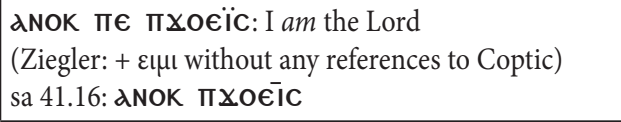 \\
\hline $44: 25$ & 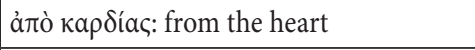 & Єво入 $2 \bar{M}$ пєҮ $2 \mathrm{HT}$ : from their heart (> Ziegler) \\
\hline $44: 25$ & $\varphi \rho v^{\prime} \mu o v \varsigma$ : the wise & $\begin{array}{l}\text { NN€ICAB€€Y: lit. these wise ones } \\
\text { (> Ziegler) sa 41.16: } \bar{N} \bar{N} C d B € € Y\end{array}$ \\
\hline $45: 3$ & 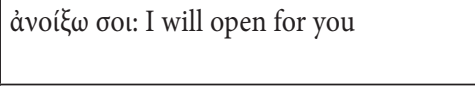 & $\begin{array}{l}\text { †NגOYWN MMOOY NaK: } \\
\text { I will open them for you (> Ziegler) }\end{array}$ \\
\hline $45: 3$ & 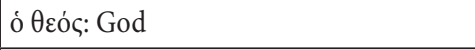 & пєКNOYT€: your God (Ziegler: + бov Sa) \\
\hline $45: 6$ & 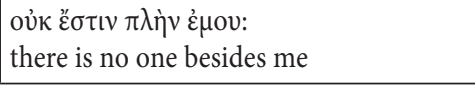 & $\begin{array}{l}\text { MN } \sigma € \mathrm{NB} \lambda \lambda \lambda \mathrm{\lambda l} \text { : there is no other besides me } \\
(\text { Ziegler: }+\varepsilon \tau \iota \mathrm{Co})\end{array}$ \\
\hline
\end{tabular}


TOMASZ BĄK

\begin{tabular}{|c|c|c|}
\hline 45:7 & 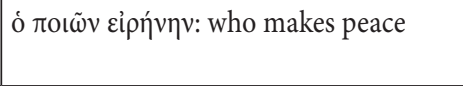 & $\begin{array}{l}\text { dNOK חє†P€ N†PHNH: } \\
\text { I am the one who makes peace (> Ziegler) }\end{array}$ \\
\hline $45: 8$ & $\dot{\varepsilon} \gamma \omega \dot{~ \varepsilon i ̉ \mu ı ~ к u ́ p ı o c: ~ I ~ a m ~ t h e ~ L o r d ~}$ & + пNOYT€: God (Ziegler: + o $\theta \varepsilon \circ \varsigma$ Co) \\
\hline 45:9 & 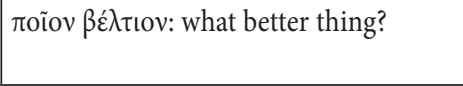 & 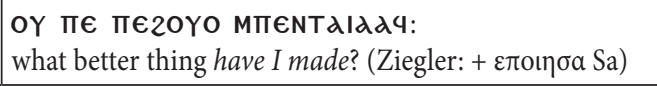 \\
\hline $45: 9$ & 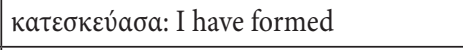 & גIT גмї̈K: I have formed you (Ziegler: $+\sigma \varepsilon$ Co) \\
\hline $45: 9$ & $\tau \grave{\nu} \nu \gamma \tilde{\eta} v$ : the earth & 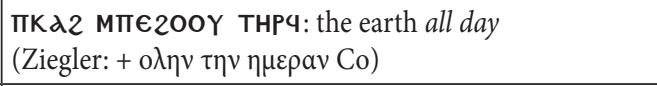 \\
\hline 45:9 & 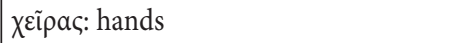 & тоо $\overline{\mathrm{TK}}$ : your hand (Ziegler: + бov Sa) \\
\hline 45:9 & 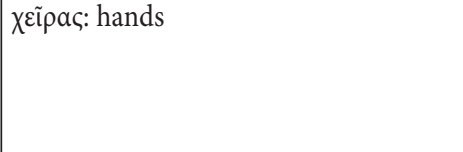 & 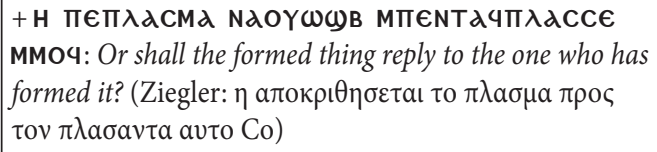 \\
\hline $45: 10$ & 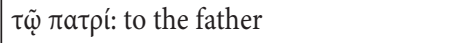 & 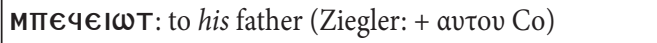 \\
\hline 45:10 & 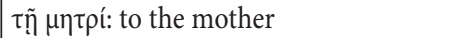 & т€чмдаY: to his mother (Ziegler: + avтov Co) \\
\hline $45: 14$ & 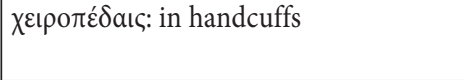 & $\begin{array}{l}\text { MாїіN€ N2OMNT ЄTOOTOY: in copper handcuffs } \\
\text { (> Ziegler) }\end{array}$ \\
\hline $45: 14$ & 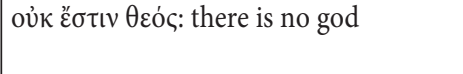 & $\begin{array}{l}\text { MN KENOYT€: there is no other god } \\
\text { (Ziegler: }+a \lambda \lambda \text { oৎ Co) }\end{array}$ \\
\hline $45: 16$ & 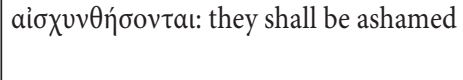 & 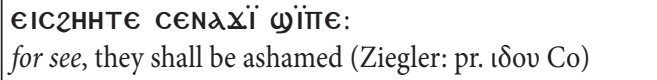 \\
\hline $45: 18$ & 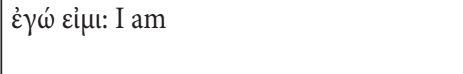 & 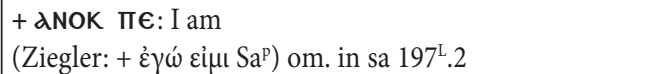 \\
\hline $45: 18$ & 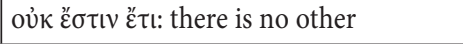 & 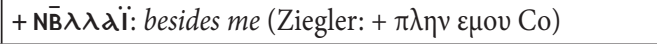 \\
\hline $45: 20$ & 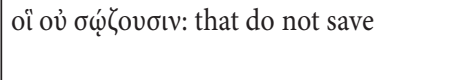 & $\begin{array}{l}\text { ETENCENATAN20OY AN: that do not save them } \\
\text { (Ziegler: + avTous without any references to Coptic) }\end{array}$ \\
\hline $45: 21$ & $\pi \alpha ́ \rho \varepsilon \xi \xi \dot{\varepsilon} \mu o v:$ except me & 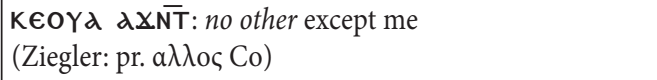 \\
\hline $45: 22$ & $\tau \tilde{\eta} \varsigma \gamma \tilde{\eta} \varsigma$ : of the earth & 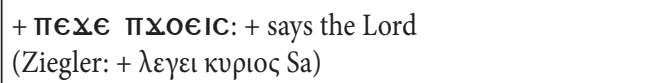 \\
\hline $45: 23$ & 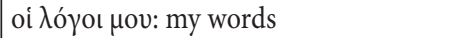 & pr. $а \gamma \omega$ (Ziegler: pr. каı Co) \\
\hline
\end{tabular}

Table 2. Omissions in the Coptic text

\begin{tabular}{|c|c|c|}
\hline $44: 6$ & kaí & lit. om. in sa 52 (> Ziegler) \\
\hline $44: 7$ & $\kappa a i^{2}$ & lit. om. in sa 52 (> Ziegler) \\
\hline $44: 8$ & oủk & om. in sa 52 (> Ziegler) \\
\hline $44: 8$ & тótع: formerly & om. in sa 52 (> Ziegler) \\
\hline $44: 10$ & кaí & lit. om. in sa 52 (> Ziegler) \\
\hline
\end{tabular}


A CRITICAL EDITION AND PHILOLOGICAL ANALYSISOF THE TEXT OF ISA 44:6-45:25

\begin{tabular}{|c|c|c|}
\hline $44: 12$ & 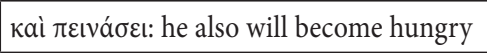 & 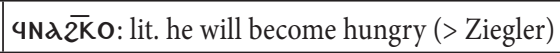 \\
\hline 44:13 & $\mathrm{kai}^{2}$ & lit. om. in sa 52 (> Ziegler) \\
\hline $44: 19$ & 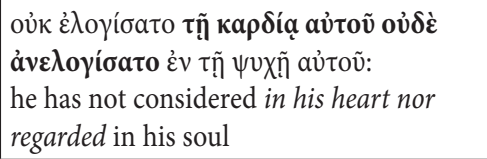 & 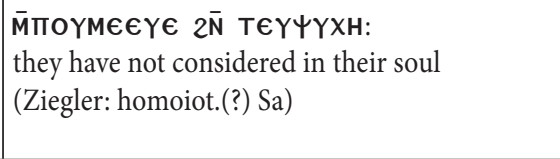 \\
\hline $44: 25$ & kai $i^{2}$ & lit. om. in sa 52 (> Ziegler) \\
\hline $45: 3$ & kai & lit. om. in sa 52 (> Ziegler) \\
\hline $45: 6$ & 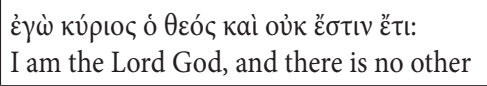 & om. in sa 52 (> Ziegler) \\
\hline $45: 11$ & 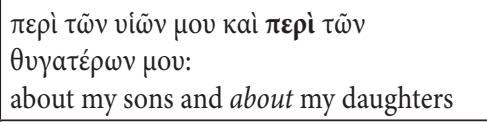 & 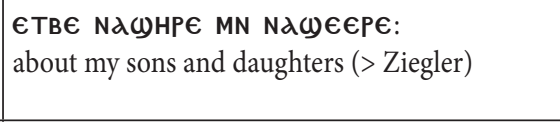 \\
\hline $45: 14$ & 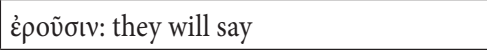 & om. in sa 52 (observed by Ziegler) \\
\hline $45: 18$ & $\kappa^{\prime} i^{1}$ & lit. om. in sa 52 (> Ziegler) \\
\hline $45: 20$ & $\omega \varsigma$ : as if & om. in sa 52 (observed by Ziegler) \\
\hline $45: 23$ & 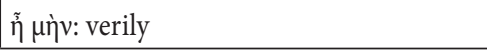 & om. in sa 52 (> Ziegler) \\
\hline
\end{tabular}

Table 3. Changes of words

\begin{tabular}{|c|c|c|}
\hline 44:11 & $\kappa \omega \varphi$ ó: [they are] mute & мєүсштм̄: they cannot hear (> Ziegler) \\
\hline 44:12 & 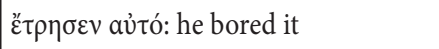 & дЧТА२О५ ЁРдТЧ: lit. he put it (> Ziegler) \\
\hline $44: 13$ & $\dot{\varepsilon} \kappa \lambda \varepsilon \xi \xi a ́ \mu \varepsilon v o \varsigma:$ having chosen & дчс $\omega \lambda \bar{\Pi}$ : having cut (> Ziegler) \\
\hline $44: 15$ & 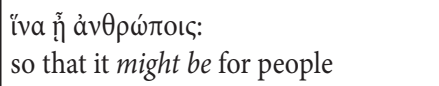 & 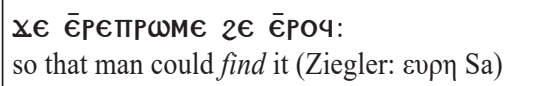 \\
\hline $44: 23$ & ò $\theta \varepsilon o ́ c:$ God & 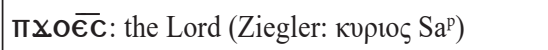 \\
\hline $44: 28$ & Kúpw: to Cyrus & 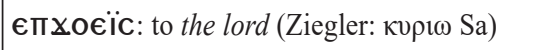 \\
\hline $45: 1$ & 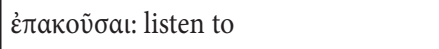 & сштт̄: to hear (> Ziegler) \\
\hline $45: 10$ & kai & H: or (Ziegler: $\eta \mathrm{Sa}$ ) \\
\hline $45: 13$ & av̉tóv: him & ПТРPO: the king (Ziegler: $\beta \alpha \sigma i \lambda \varepsilon \alpha$ Co) \\
\hline $45: 17$ & 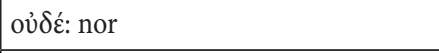 & aYw: lit. and (> Ziegler) \\
\hline $45: 18$ & 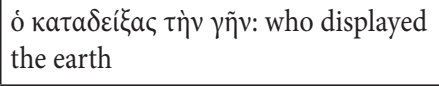 & 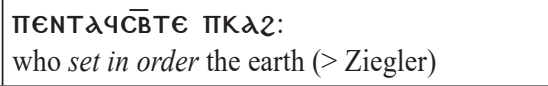 \\
\hline $45: 20$ & $\gamma \lambda \hat{\mu} \mu \mu \alpha:$ engraved figure & 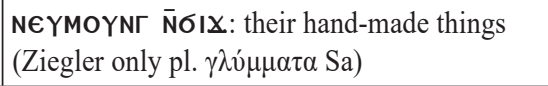 \\
\hline $45: 21$ & 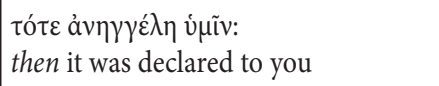 & $\begin{array}{l}\text { NTAYXOOY NHTN TNAY: } \\
\text { when it was declared to you (Ziegler: } \pi 0 \tau \varepsilon \mathrm{Sa} \text { ) }\end{array}$ \\
\hline $45: 22$ & $\sigma \omega \theta \eta ́ \sigma \varepsilon \sigma \theta \varepsilon:$ you shall be saved & TגР€T€TN $\omega \bar{N} 2:$ you shall live (> Ziegler) \\
\hline
\end{tabular}




\begin{tabular}{|c|c|c|}
\hline & 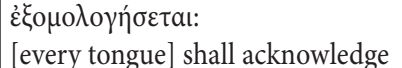 & WPK: [every tongue] shall swear (Ziegler: o \\
\hline
\end{tabular}

Table 4. Changes of prepositions

\begin{tabular}{|c|c|c|}
\hline $44: 11$ & ö $\theta \varepsilon v$ (adverb): from where & $2 \overline{\mathrm{M}}:$ in $(>$ Ziegler $)$ \\
\hline $44: 11$ & àmò ả $v \theta \rho \omega ́ \pi \omega v$ : from among men & ЄРФм€: [cannnot hear] men (> Ziegler) \\
\hline 44:14 & 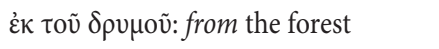 & 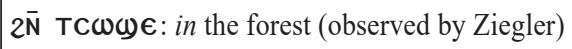 \\
\hline 44:19 & $\dot{\varepsilon} \pi \mathrm{i} \tau \tilde{\omega} \nu$ ả $v \theta \rho a ́ k \omega \nu$ aủंoṽ: on its coals & 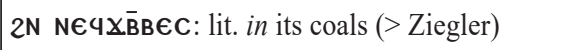 \\
\hline $44: 23$ & Ėv aùtoĩc: in them & zïwoY: lit. on them (> Ziegler) \\
\hline $44: 25$ & $\begin{array}{l}\sigma \eta \mu \varepsilon \tilde{\alpha} \alpha \dot{\varepsilon} \gamma \gamma \alpha \sigma \tau \rho \mu v ́ \theta \omega v \text { : } \\
\text { lit. the signs of ventriloquists }\end{array}$ & 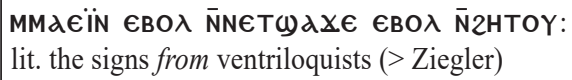 \\
\hline
\end{tabular}

Table 5. Changes of articles

\begin{tabular}{|c|c|c|}
\hline $44: 7$ & 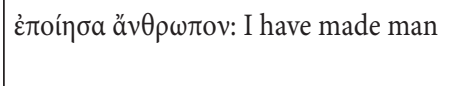 & $\begin{array}{l}\text { дїтдмїє прФмє: lit. I have made the man } \\
\text { (Ziegler: pr. } \tau \text { ov without any references to Coptic) }\end{array}$ \\
\hline $44: 23$ & $\theta \varepsilon \mu \varepsilon ́ \lambda ı \alpha$ : foundations & $\overline{\mathbf{N}} \mathrm{C} \overline{\mathrm{N}} \mathrm{T} \epsilon$ : lit. the foundations (Ziegler: pr. $\tau \alpha \mathrm{Co}$ ) \\
\hline $44: 23$ & 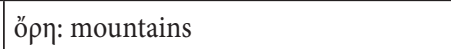 & NTOOY: lit. the mountains (Ziegler: pr. $\tau \alpha \mathrm{Co}$ ) \\
\hline $45: 12$ & $\gamma \tilde{\eta} v:$ earth & $\begin{array}{l}\text { חKג2: the earth } \\
\text { (Ziegler: pr. } \tau \eta \nu \text { without any references to Coptic) }\end{array}$ \\
\hline $45: 15$ & $\sigma \omega \tau$ tŕp: Savior & псштнр: lit. the Savior (Ziegler: pr. о Co) \\
\hline $45: 19$ & кúpısc: lit. Lord & пхоєї: the Lord (> Ziegler, see the commentary) \\
\hline
\end{tabular}

Table 6. Changes in word order

\begin{tabular}{|c|c|c|}
\hline $44: 6$ & 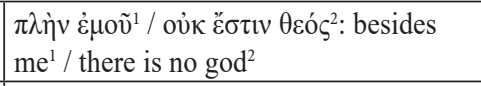 & $\bar{M} \bar{N} K \in N O Y T \epsilon^{2} / N \bar{N} \bar{\lambda} \lambda d \ddot{I}^{1}$ (> Ziegler) \\
\hline $44: 7$ & 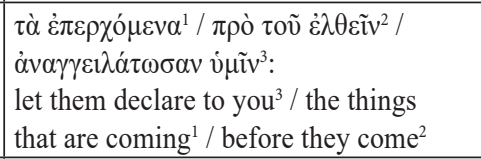 & 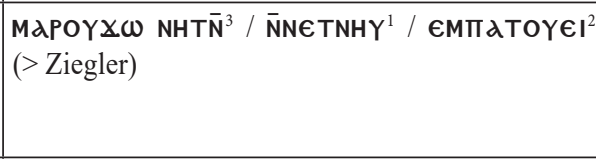 \\
\hline $44: 12$ & 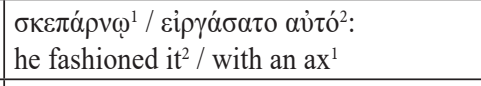 & 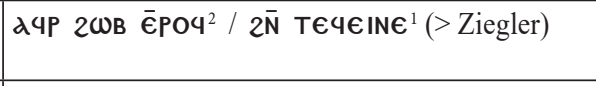 \\
\hline $44: 13$ & 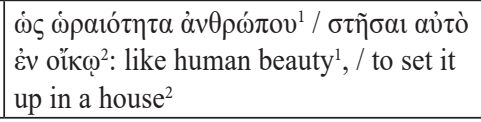 & 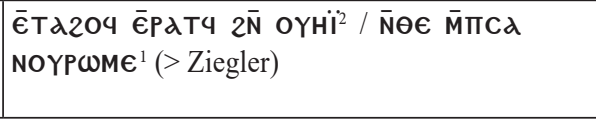 \\
\hline
\end{tabular}


A CRITICAL EDITION AND PHILOLOGICAL ANALYSISOF THE TEXT OF ISA 44:6-45:25

\begin{tabular}{|c|c|c|}
\hline $44: 14$ & 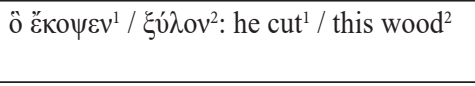 & $\begin{array}{l}\pi € \mid \omega \epsilon^{2} / \epsilon N T d \varphi \omega \lambda d \alpha \overline{\mathbf{q}}^{1} \\
\text { (observed by Ziegler, without any references to Coptic) }\end{array}$ \\
\hline 44:17 & $\begin{array}{l}\theta \varepsilon o ́ \varsigma \\
\operatorname{god}^{1}\end{array}$ & NTOK $^{3} / \pi \epsilon^{2} /$ ПגNOYT€ ${ }^{1}$ (> Ziegler) \\
\hline 44:22 & 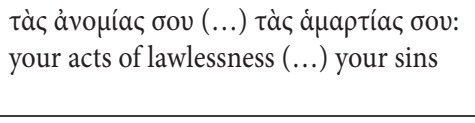 & 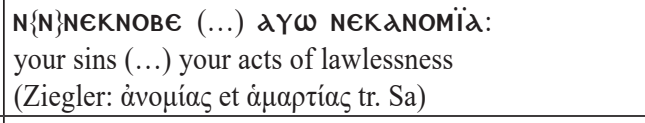 \\
\hline $45: 11$ & 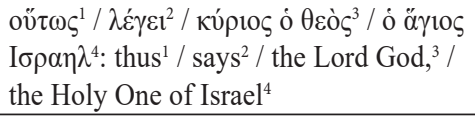 & 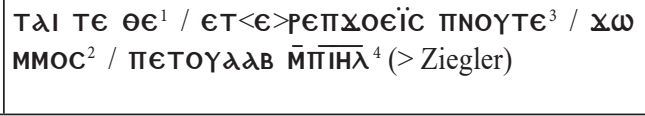 \\
\hline
\end{tabular}

Table 7. Semantic changes

\begin{tabular}{|c|c|c|}
\hline 44:6 & 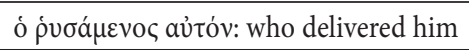 & חєNTA 4Nג2M€K: who delivered you (Ziegler: $\sigma \varepsilon$ ) \\
\hline 44:8 & 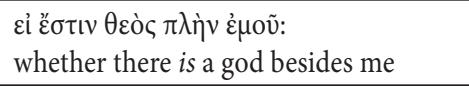 & $\begin{array}{l}\mathbf{X} \in \mathbf{N} \in \mathbf{Y N} \text { KENOYT€ } \overline{\mathbf{N}} \overline{\mathrm{B}} \lambda \lambda d \ddot{\mathrm{I}}: \\
\text { whether there was a god besides me (> Ziegler) }\end{array}$ \\
\hline 44:10 & $\theta$ cóv: god & N̄NENOYT€: gods (Ziegler: $\theta$ couৎ Co) \\
\hline 44:15 & àv$\theta \rho \omega ́ \pi$ orc: for people & пршмє: the man (Ziegler: $\alpha v \theta \rho \omega \pi о \varsigma \mathrm{Sa}$ ) \\
\hline 44:15 & 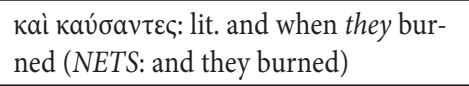 & 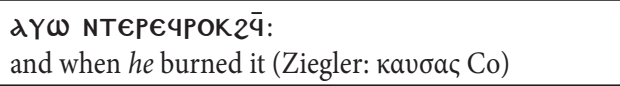 \\
\hline 44:15 & 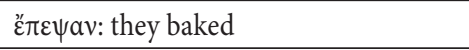 & дчпєс: he baked (Ziegler: $\varepsilon \pi \varepsilon \psi \varepsilon v$ Co) \\
\hline 44:15 & 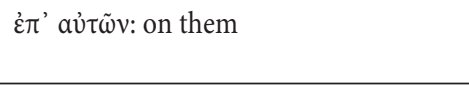 & 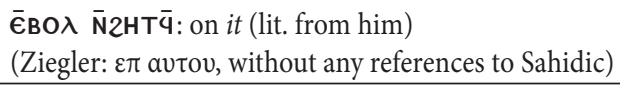 \\
\hline 44:16 & крع́ac: meat (sg.) & $2 \overline{\mathrm{N}} \lambda 4:$ meats (pl.) (> Ziegler) \\
\hline 44:16 & ỏ $\tau \tau \dot{\eta} \sigma \alpha \varsigma$ : after roasting & $\begin{array}{l}\text { Ачб€б: he roasted } \\
\text { (Ziegler: } \omega \pi \tau \eta \sigma \varepsilon v, \text { without any references to Coptic) }\end{array}$ \\
\hline 44:17 & 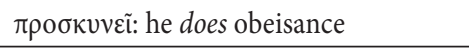 & дчпд2Тч: he did obeisance (> Ziegler) \\
\hline 44:17 & 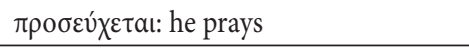 & $\lambda \varphi \omega \lambda \boldsymbol{H} \lambda$ : he prayed (> Ziegler) \\
\hline 44:19 & 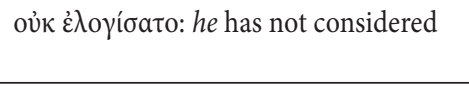 & 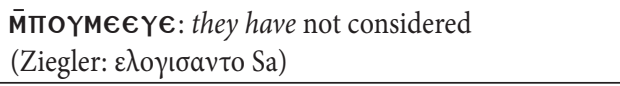 \\
\hline 44:19 & 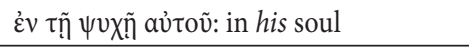 & 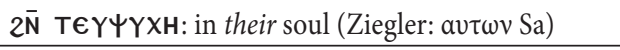 \\
\hline 44:19 & 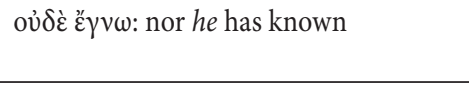 & $\begin{array}{l}\text { oү } \Delta € \overline{\text { м} п о \gamma є і ̈ м €: ~ n o r ~ t h e y ~ h a v e ~ k n o w n ~} \\
\text { (Ziegler: } \varepsilon \gamma \nu \omega \sigma a v \mathrm{Sa} \text { ) }\end{array}$ \\
\hline 44:19 & 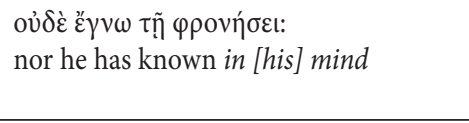 & 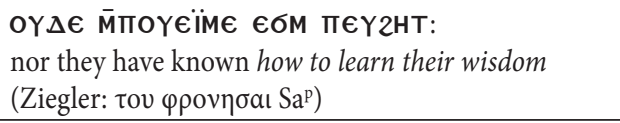 \\
\hline 44:19 & 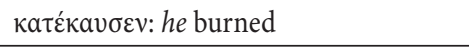 & 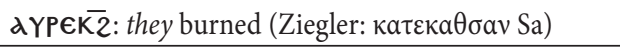 \\
\hline 44:19 & $\varepsilon ૈ \pi \varepsilon \psi \varepsilon v:$ he baked & dүחєC: they baked (Ziegler: $\varepsilon \pi \varepsilon \psi a \nu ~ S a)$ \\
\hline 44:19 & крع́ac: meat & $2 \overline{\mathrm{N}} \lambda \mathrm{\varphi}_{\text {: meats }}$ (> Ziegler) \\
\hline 44:19 & 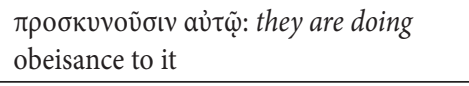 & 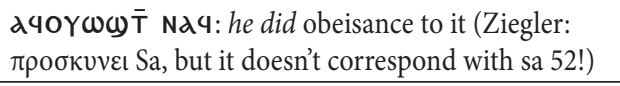 \\
\hline $44: 20$ & $\pi \lambda \alpha v \tilde{\omega} \nu \tau \alpha$ l: they are going astray & $\overline{\mathbf{q}} \Pi \lambda \lambda \mathrm{N} \alpha$ : lit. he is going astray (> Ziegler) \\
\hline
\end{tabular}


TOMASZ BĄK

\begin{tabular}{|c|c|c|}
\hline 44:20 & 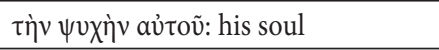 & 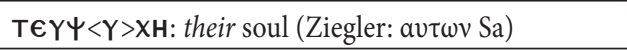 \\
\hline $44: 25$ & 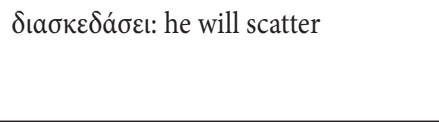 & $\begin{array}{l}€ N T d \varphi \times \epsilon \in P €: \text { who scattered } \\
\text { (Ziegler: } \delta\llcorner\varepsilon \sigma \kappa \varepsilon \delta \alpha \sigma \varepsilon(v) \text { without any references } \\
\text { to Coptic) }\end{array}$ \\
\hline 44:26 & 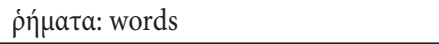 & 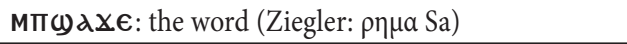 \\
\hline 44:28 & 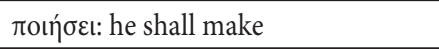 & 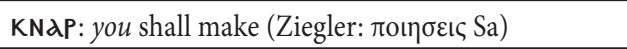 \\
\hline $45: 2$ & 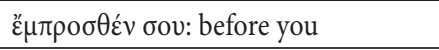 & 2д тєч2Н: before him (Ziegler: avтоv Co) \\
\hline $45: 7$ & 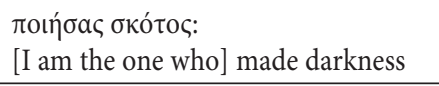 & $\begin{array}{l}\text { дїТдмІє ПКдКє: } \\
\text { I made darkness (Ziegler: pr. } \varepsilon \gamma \omega \text { Co) }\end{array}$ \\
\hline $45: 9$ & 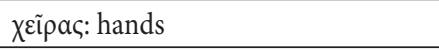 & 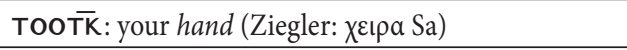 \\
\hline $45: 10$ & 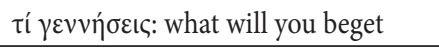 & д2РОК Кхпо ммої: what do you beget? (> Ziegler) \\
\hline $45: 11$ & I $\sigma \rho a \eta \lambda:$ Israel & $\begin{array}{l}\overline{\mathrm{M}} \overline{\Pi \mathrm{IH} \lambda} \text { : of Israel } \\
\text { (Ziegler: pr. tov without any references to Coptic) }\end{array}$ \\
\hline $45: 17$ & 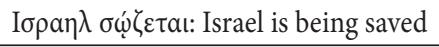 & 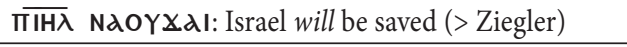 \\
\hline $45: 20$ & 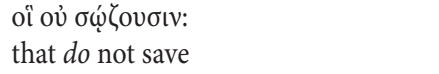 & $\begin{array}{l}€ T € \text { NCENATAN20OY AN: } \\
\text { that will not vivify them (> Ziegler) }\end{array}$ \\
\hline
\end{tabular}

Table 8. Greek words in the Coptic text

\begin{tabular}{|c|c|c|}
\hline $44: 26$ & ä $\gamma \gamma \varepsilon \lambda о \varsigma$ & дГгє \\
\hline $45: 13$ & aỉ $\mu \alpha \lambda \omega \sigma i ́ a$ & 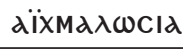 \\
\hline $44: 9$ & $\dot{a} \lambda \lambda \alpha^{\prime}$ & $d \lambda \lambda d$ \\
\hline $44: 22$ & àvoría & dNOMİd \\
\hline $44: 22 ; 45: 15$ & $\gamma \dot{\alpha} \rho$ & $\Gamma d P$ \\
\hline $44: 17 ; 45: 4$ & $\delta \dot{\varepsilon}$ & $\Delta \epsilon$ \\
\hline 45:8 (2x) and v. 13 & 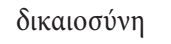 & $\Delta \ddot{K} \alpha$ גIOCYNH \\
\hline $45: 1$ & 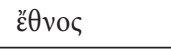 & $2 \epsilon \Theta N O C$ \\
\hline $45: 7$ & 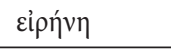 & TPHNH \\
\hline $45: 14$ & 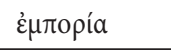 & м̄TOPId \\
\hline $44: 23 ; 45: 8$ & 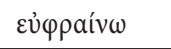 & $€ Y$ ФРАN€ \\
\hline 45:9.10 & $\ddot{\eta}$ & H \\
\hline $44: 21.23 ; 45: 4$ & $\operatorname{Ia\kappa } \omega \beta$ & $I A K \omega B$ \\
\hline $44: 26.28$ & 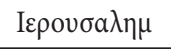 & $\theta \mid \overline{\lambda H M}$ \\
\hline $44: 26$ & 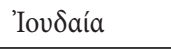 & toYsdld \\
\hline $\begin{array}{l}\text { 44:6.21 (2x) and v. } 23(2 \mathrm{x}) \\
45: 3.4 .11 .15\end{array}$ & I $\sigma \rho \propto \eta \lambda$ & $\overline{\Pi \mathrm{IH} \lambda}$ \\
\hline $44: 9$ & 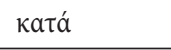 & KdTd \\
\hline $45: 9(2 x)$ & 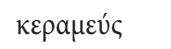 & КєРдмєYС \\
\hline
\end{tabular}


A CRITICAL EDITION AND PHILOLOGICAL ANALYSISOF THE TEXT OF ISA 44:6-45:25

\begin{tabular}{|c|c|c|}
\hline 45:1 & Kũ pos & KYPOC \\
\hline $45: 13$ & 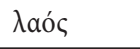 & $\lambda d O c$ \\
\hline $45: 9$ & $\mu \eta ́$ & MH \\
\hline $45: 2$ & 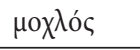 & MOX $\lambda O C$ \\
\hline $44: 19 ; 45: 13$ & 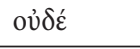 & OY $\Delta \epsilon$ \\
\hline 44:20 & $\pi \lambda \alpha v \alpha \dot{\alpha} \omega$ & $\Pi \lambda \lambda \alpha \lambda d$ \\
\hline $44: 8.10 .21 .24$ & $\pi \lambda \alpha ́ \sigma \sigma \omega$ & $\Pi \lambda \lambda \alpha c c \epsilon$ \\
\hline $44: 26 ; 45: 1.13$ & 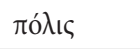 & подіс \\
\hline 44:6; 45:13.14 & $\sigma \alpha \beta \alpha \omega \theta^{179}$ & $C \lambda B d \omega \theta$ \\
\hline $44: 23$ & $\sigma \alpha \lambda \pi i \zeta \omega$ & 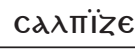 \\
\hline $45: 14$ & $\sum \varepsilon \beta \omega t v$ & CגBdEIN \\
\hline $45: 15$ & $\sigma \omega \tau \eta \dot{\rho} \rho$ & CWTHP \\
\hline $54: 1$ & Xрıбтós & ХРнСтос \\
\hline $44: 19.20$ & $\psi v \times \eta^{\prime}$ & భOYXH \\
\hline
\end{tabular}

\section{The Analysis of Selected Philological Questions Found in Isa 44:6-45:25}

The last part of the paper analyses the more difficult philological questions found in Isa 44:6-45:25 concerning two areas. Firstly, they can result from differences between the Sahidic manuscripts, which has been indicated in the critical apparatus of the Coptic text. Secondly, they may relate to the way of reading and translating the Greek text of the Septuagint into the Coptic language. The philological issues requiring commentary can be found in the following verses:

\section{Isa 44:8}

Looking at the first words of the verse in the Coptic rendering we can see not only an omission of the Greek negation ov̉к, but also a slightly altered punctuation, affecting a different understanding of the text. In the Greek LXX we read

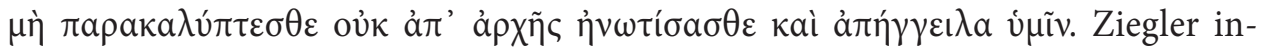
troduces a dot after $\pi \alpha \rho \alpha \kappa \alpha \lambda u ́ \pi \tau \varepsilon \sigma \theta \varepsilon$, and a question mark after $\dot{u} \mu \tilde{v}$ so as to read, "Do not cover yourselves; did you not give ear from the beginning, and I declared it to you?" The Coptic text omits ov̉к, connecting å $\pi^{\prime}$ à $\rho \chi \tilde{\eta} \varsigma$ with

179 The term $\sigma \alpha \beta \alpha \omega \theta$ originally comes from Hebrew. 


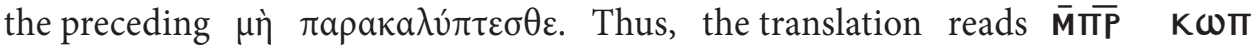
Хiin $\overline{\mathrm{N}} \boldsymbol{O} \mathrm{OPT}$, which can be rendered as "Do not cover yourselves from the beginning." The next part of the Coptic verse is not a question but a statement

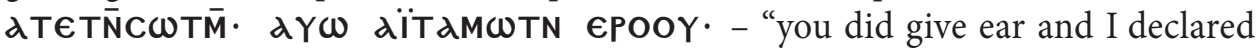
it to you." Without looking at the Coptic punctuation, we could also read: "Do not cover yourselves! From the beginning you did give ear and I declared to you."

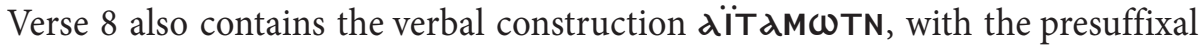
form of the verb TaMO ${ }^{180}$ However, its correct version should have a short $\mathbf{O}$ instead of the vowel $\omega$. So the construction should be: дїтдмотN. The second vowel in the presuffixal form $T \lambda \mathbf{M} \omega=$ seems to characterise our manuscript sa 52 since it can also be found in Isa 5:5; 21:10; 33:14; 43:12.

Ziegler's critical apparatus shows that the Sahidic manuscripts have $\eta \kappa o v \sigma \alpha v$ in

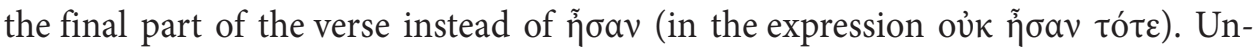
fortunately, we do not know to which Coptic manuscript this observation refers. Among the available witnesses, Isa 44:8 can only be found in manuscript sa 52, which

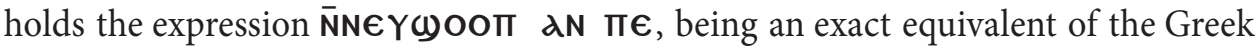

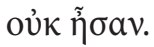

\section{Isa 44:9}

The Greek text, in accordance with its verse division, begins with the words of

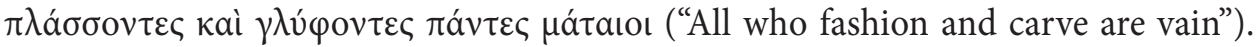

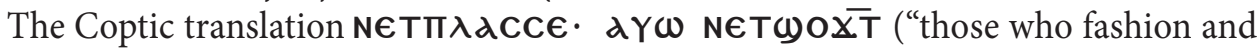

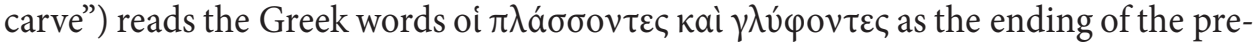

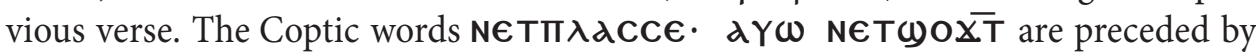
the particle $\overline{\mathbf{N}} \sigma \mathbf{I}$, introducing the subject to the preceding expression $\overline{\mathbf{N}} \mathbf{N} \boldsymbol{Y} \boldsymbol{\omega} \mathrm{OO}$ aN $\pi \boldsymbol{~} \boldsymbol{\epsilon}$. Thus, the Coptic translator considered verse 8 to be slightly longer and

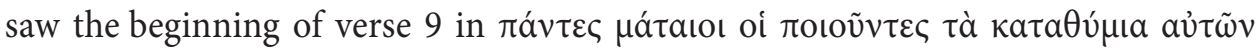
("All who do the things that are in their minds are vain").

\section{Isa 44:10}

The beginning of the verse is another example of a different syntactic combination

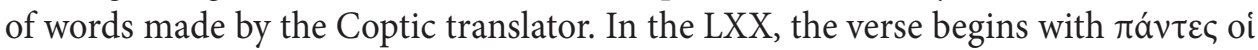

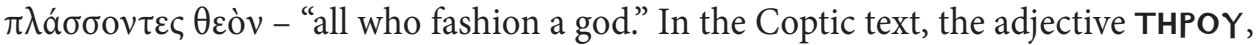
as an equivalent of the Greek $\pi \dot{v} v \tau \varepsilon \varsigma$, is syntactically connected with the ending of

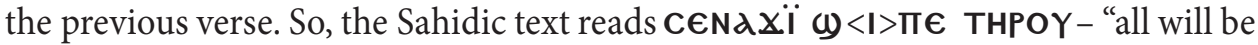
put to shame." Then the particle $\overline{\mathbf{N}} \boldsymbol{\sigma} \mathrm{I}$ is introduced followed by an explanation who those THPOY are, i.e. N€TM $\lambda$ dCC€ NNENOYT€ - "all who fashion gods." Summing 
up, the Greek text reads "But they will be put to shame, all who fashion a god," while the Coptic text proposes "But they all will be put to shame, who fashion gods."

\section{Isa 44:11}

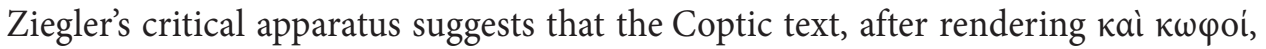

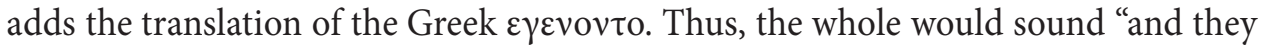

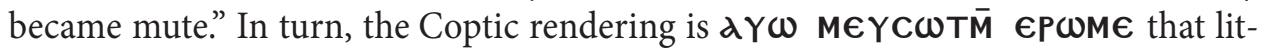
erary means "and they cannot hear people." We do not know which manuscripts were referred to by Ziegler, but his suggestion is not consistent with the text of our manuscript sa 52 .

At the end of the verse there is the word MapoY $\omega \lambda$ C. Its first part must be the base of jussive conjugation in the 3rd pers. plural MapoY-. The second part is

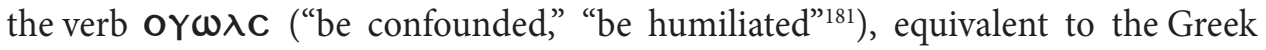

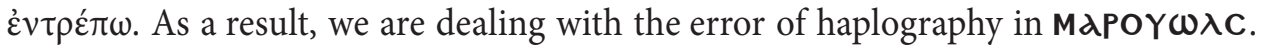

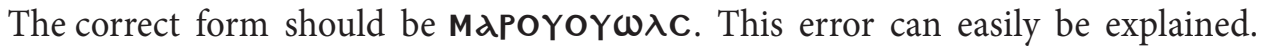
The letters of the first part of the word (MAPOY-) complete the column on page $\overline{\mathrm{CB}}$, while the next letters $(-\boldsymbol{\omega} \lambda \mathbf{C})$ begin the column on page $\overline{\mathbf{Y}} \mathbf{\Gamma}$. So the scribe, after having written the letters -OY- on the previous page, did not notice that he should have repeated them on the following page. The reading MdPOY $<0 \gamma>\omega \lambda \mathrm{C}$ has been used in our edition.

\section{Isa 44:12}

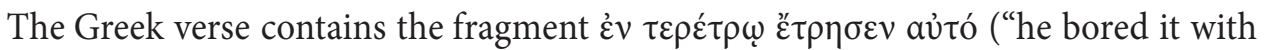

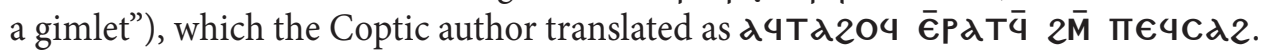
However, the meaning of the Coptic construction $\mathbf{T} \mathbf{2} 2 \mathbf{O}=\mathbf{\epsilon P \lambda T}=$ does not refer to

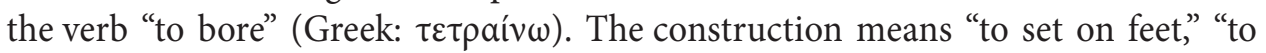
make to stand" and "to establish." ${ }^{182}$ Consequently, the literal rendering in Coptic is "he made it stand with his gimlet." Perhaps to set up the statue of a deity, it was necessary to bore a hole in it. The Greek text, speaking only about boring a statue may raise a question about the purposefulness of such an action. On the other hand, the Coptic translation would assume the reader's knowledge that a gimlet could be used to set up a sculpture. Since this explanation is only a hypothesis, and Ziegler does not notice any difference between the Greek and Coptic texts in his critical apparatus, our translation into English follows the NETS version, i.e. "he bored it with a gimlet."

181 Crum, Coptic Dictionary, 477b.

182 Crum, Coptic Dictionary, 456a. 


\section{Isa 44:13}

At the very beginning of the verse we can note a difference between the text of the LXX and the Coptic translation. The Greek text reads $\dot{\kappa} \kappa \lambda \varepsilon \xi \dot{\alpha} \mu \varepsilon v o \varsigma^{183}$ ("having chosen"), and the Coptic rendering has $\alpha 4 c \omega \lambda \bar{\pi}$ ("having cut"184). The change of

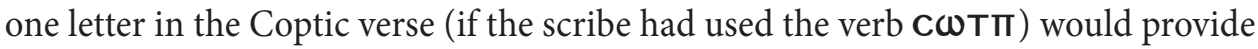
a faithful translation of the Greek text. Thus, it could have been a translator's mistake or copyist's error and not an intended textual change.

\section{Isa 44:16}

This verse contains the word $\bar{M} \Pi \mathrm{K}$ W2T, which literary means "of the fire," not matching the context. The Greek version $\dot{\varepsilon} v \pi \dot{\rho} \rho$ suggests that the correct form should be $2 \bar{M} \Pi \Pi \omega \overline{2 T}$. This reading also occurs in manuscript sa 41.16. Our edition has $<2>\bar{M} \pi K \omega 2 T$.

\section{Isa 44:19}

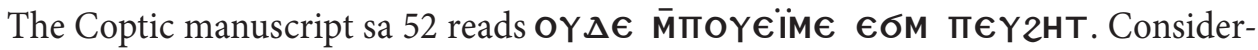
ing that the construction $\sigma \mathbf{N}-2 \mathrm{HT}$ means "to find heart" and "to learn wisdom," 185 the text of our manuscript should be rendered "nor they have known how to learn their wisdom." This version agrees with the Greek manuscript number 538, which reads tov $\varphi \rho$ p $\eta \sigma a$ s. Ziegler indicates that perhaps referring to our manuscript, this reading only occurs in some Sahidic manuscripts. ${ }^{186}$

Considering manuscript sa 41.16 we can observe the slightly different reading $2 \overline{\mathbf{M}}$ пєҮ $2 \mathrm{HT}$, i.e. literary "in their heart." It is closer to the Greek text that has

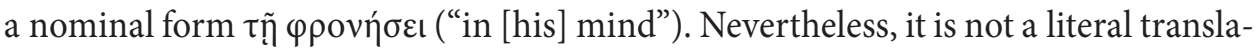
tion. Since the Coptic $2 \bar{M}$ пє $\mathbf{Y} 2 \mathbf{H T}$ would have been a literal rendering of the Greek

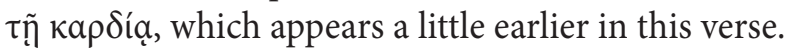

We can note that the subject was changed, which is hardly explainable in this verse. The first part has a 3rd pers. plural form, and then from the words $\alpha 4 \sigma € \sigma 2 \bar{N} \lambda \varphi$ ("he roasted meats"), the subject is in a 3rd pers. sing. masculine form. This phenomenon can be noted both in our manuscript sa 52 and in sa 41.16. Ziegler's suggestion that Sahidic texts have the plural $\varepsilon \varphi$ ayov (instead of the singular $\tilde{\varepsilon} \varphi a \gamma \varepsilon v$ ) is not supported by our manuscripts.

\footnotetext{
183 According to the division of the text, this verb belongs to v. 12.

184 Cf. Crum, Coptic Dictionary, 330b.

185 Crum, Coptic Dictionary, 820b.

186 In his critical apparatus he uses Sap, which means "ein Teil der Bruchstücke" (Ziegler, Septuaginta, 373).
} 
Isa 44:22

The Coptic manuscripts sa 52 and sa 41.16 contain two different words: KTOK and $[\mathrm{KO}] \overline{\mathrm{TK}}$. The first one is a presuffixal form of the verb $\mathrm{K} \boldsymbol{\omega T \epsilon}$. The other is a presuffixal form of the verb KTO. Both verbs have similar meanings: "to turn" and "to return" and are the Coptic translations of the Greek $\dot{\varepsilon} \pi \iota \tau \tau \rho \dot{\varepsilon} \varphi \omega .{ }^{187}$ So they can be treated as synonyms.

\section{Isa 44:28}

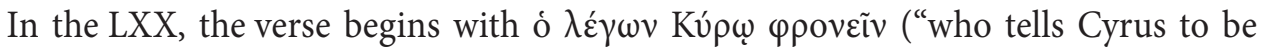
wise"). This refers to Cyrus the Great, who in 538 B.C. issued a decree allowing the Israelites to return from Babylonian captivity to their country. At this point, the Coptic translation introduces an unexpected change, reading пєТх $\omega$ ммос

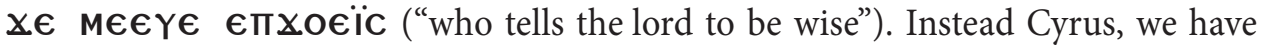
the word $€$ $\Pi \mathbf{x} \mathbf{\epsilon}$ ic ("to the lord"), according to one of the Greek manuscripts, namely manuscript 534 reading $\kappa v \rho \mathrm{\omega} \omega$. Because of the context, the term "lord" cannot refer to God. It is hard to find a motive explaining an intended change from Cyrus to "lord." Most likely, there was a simple mistake (iotacism). The Coptic translator may already have used the revised Greek text.

\section{Isa $45: 3$}

The dots, introduced by the author of the Coptic manuscript, suggest a slightly different division of the initial part of this verse. In Ziegler's LXX, we can read $\delta \omega \sigma \omega \sigma o$ o

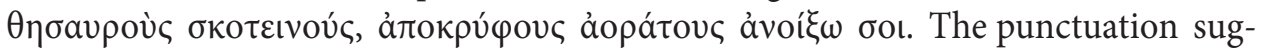
gests the reading "I will give you dark treasures; hidden, unseen ones I will open for you." Manuscript sa 52: Td† NdK N̄ $2 \epsilon N d 2 \omega \omega P$ NKdK€ $2 \bar{N} \bar{N} \epsilon \Theta H \Pi \cdot \epsilon N C € N d Y$ €POOY $d \mathbf{N} \cdot$ proposes an introduced division following the Coptic $2 \overline{\mathbf{N}} \overline{\mathbf{N}} \epsilon \Theta \mathrm{H} \Pi$, i.e. the Greek árokpú you dark treasures from what is secret, unseen."

\section{Isa $45: 9$}

The Coptic translation of the first part of the verse contains the additional verb $€$ IP€ ("to make," "to do"188) in its presuffixal form $\mathbf{d} \mathbf{d}=$. It corresponds to the Greek verb $\varepsilon \pi$ oı $\sigma \alpha$, occurring only in manuscript 538 . Therefore, it can be noted that the Coptic

187 Cf. Crum, Coptic Dictionary, 124a-129a.

188 Crum, Coptic Dictionary, 83a. 
text OY пє пє20YO мпєNTdIdגч (“What better thing have I made?") is a pre-

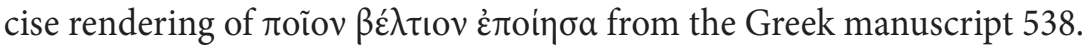

\section{Isa 45:11}

The final part of the verse contains $€$ T€N€2BHY€ NNגбIX. The Coptic should be

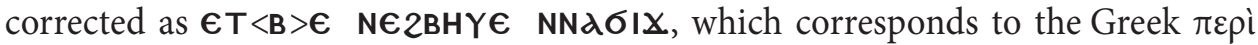
$\tau \tilde{\omega} \nu$ है $\rho \gamma \omega \nu \tau \tilde{\omega} \nu \chi \varepsilon\left\llcorner\rho \tilde{\omega} \nu \mu o v\right.$ ("concerning the works of my hands"). ${ }^{189}$

\section{Isa 45: 19}

In his critical apparatus to the final part of the verse, Ziegler suggests that at least a few Greek manuscripts as well as the Coptic text in the Sahidic dialect read the participle $\lambda \alpha \lambda \tilde{\omega} v$ with the masculine article o. His remark is not supported by manuscripts sa 52 and sa $197^{\mathrm{L}} .2$, which have $€ \mathbf{T} \mathbf{x} \boldsymbol{\omega}$, and not п€тх $\boldsymbol{\omega}$. Ziegler's note might have referred to the noun kúpıos, which the Coptic rendering reads together with the article: $\Pi \mathbf{x} \mathbf{0} \ddot{\mathrm{ic}}$.

\section{Isa 45:21}

A comparison of the Greek and Coptic texts indicates a slightly different division of the middle part of the verse. In Ziegler's edition, a dot was put after the expres-

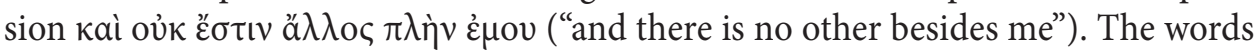

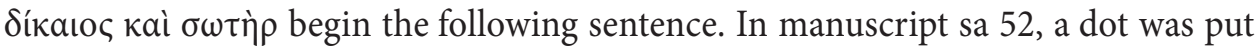
only after the word NCWTHP. ${ }^{190}$ Consequently, it is logical to read $\alpha Y \omega \bar{M} \bar{N}$ K€OY $d$

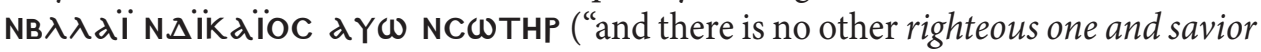
besides me").

\section{Isa 45:23}

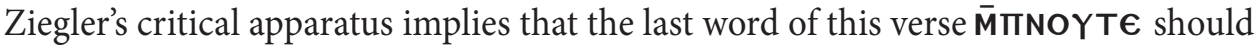
be interpreted as per deum. It is difficult to unequivocally agree with this opinion. Since $\overline{\mathbf{M}}$ - preceding the noun TNOYT€ can appear as a dative. ${ }^{191}$ The expression WPK M̄TNOYT€ simply means "to swear to God." Therefore, the Coptic $\bar{M}$ TINOYT€ agrees with the literal understanding of the Greek $\tau \tilde{\omega} \theta \varepsilon \tilde{\omega}$.

\footnotetext{
189 Cf. Crum, Coptic Dictionary, 688a.

190 Obviously, interpreting the Coptic manuscripts we should analyse all "punctuation" signs with great caution. Nevertherless, the dot after the word NCWTHP can also be seen in manuscript sa 41.17 (see Wessely, Griechische und koptische Texte, No. 220e). The sign N- before cWTHP is important, too: we have two predications with the first $\mathbf{d} \gamma \boldsymbol{\omega}$.

191 Cf. Crum, Coptic Dictionary, 529b.
} 


\section{Conclusion}

The edition of the Coptic text of Isa 44:6-45:25 on the basis of various Sahidic manuscripts, especially codex sa 52, shows the uniqueness of the manuscript from the Pierpont Morgan collection. As already noted in the second part of the paper, as much as $75 \%$ of the selected section of the Book of Deutero-Isaiah is found only in manuscript sa 52. At present, it is the only manuscript with the complete text of the Book of Isaiah, which has not yet been fully published. Hopefully, the Sahidic edition of Isa 44:6-45:25, its English translation, comparison with the Greek Septuagint and explanation of the more difficult philological issues will contribute to an even better understanding of both the message of this book of Scripture and the history of its transmission.

\section{Bibliography}

Amélineau, É., "Fragments de la version thébaine de l'Écriture (Ancien Testament)," Recueil de travaux relatifs à la philologie et à l'archéologie égyptiennes et assyriennes 9 (1887) 101-130.

Atanassova, D., "Zu den sahidischen Pascha-Lektionaren," Orientalia Lovaniensia Analecta 133/2 (2004) 607-620.

Bąk, T., "A Critical Edition and Philological Analysis of the First Chapter of Deutero-Isaiah (Isa 40) on the Basis of the Coptic Manuscript sa 52 (M 568) in Light of Other Coptic Manuscripts Written in the Sahidic Dialect and the Greek Text of the Septuagint," The Biblical Annals 9/1 (2019) 73-100 (= Isa 40).

Bąk, T., "A Critical Edition and Philological Analysis of the Second Chapter of Deutero-Isaiah (Isa 41) on the Basis of the Coptic Manuscript sa 52 (M 568) in Light of Other Coptic Manuscripts Written in the Sahidic Dialect and the Greek Text of the Septuagint," The Biblical Annals 10/1 (2020) 63-91 (= Isa 41).

Bąk, T., "A Critical Edition and Philological Analysis of the Text of Isa 42:1-44:5 on the Basis of the Coptic Manuscript sa 52 (M 568) and Other Coptic Manuscripts Written in the Sahidic Dialect of Coptic and the Greek Text of the Septuagint," The Biblical Annals 10/2 (2020) 225-260 (= Isa 42:1-44:5).

Bąk, T., "Koptyjski przekład Iz 1-39 w manuskrypcie sa 52 (M 568) z VIII w.", The Biblical Annals 5/2 (2015) 289-305.

Bąk, T., Il Proto-Isaia in copto-saidico. Edizione critica sulla base di sa 52 (M 568) e di altri testimoni. Estratto della dissertazione per il Dottorato in Studi dell'Oriente Antico (Roma: Pontificio Instituto Biblico, Facoltà Orientalistica 2014).

Ciasca, A., Sacrorum Bibliorum fragmenta copto-sahidica Musei Borgiani iussu et sumptibus S. Congregationis de Propaganda Fide studio P. Augustini Ciasca ordinis Eremitarum S. Agostini edita (Roma: Typis S. Congregationis de Propaganda Fide 1885-1889) I-II.

Crum, W.E., Catalogue of the Coptic Manuscripts in the British Museum (London: British Museum 1905). 
Crum, W.E., A Coptic Dictionary (Oxford: Clarendon Press 1939) (reprint: Eugene, OR: Wipf \& Stock 2005).

Crum, W.E. - Winlock, H.E., The Monastery of Epiphanius at Thebes. I. The Archeological Material by H.E. Winlock. The Literary Material by W.E. Crum (New York: Metropolitan Museum of Art 1926).

Crum, W.E. - Evelyn White, H.G., The Monastery of Epiphanius at Thebes. II. Coptic Ostraca and Papyri Edited with Translations and Commentaries by W.E. Crum. Greek Ostraca and Papyri Edited with Translations and Commentaries by H.G. Evelyn White (New York: Metropolitan Museum of Art 1926).

Depuydt, L., Catalogue of Coptic Manuscripts in the Pierpont Morgan Library (Corpus van verluchte handschriften 4. Oriental Series 1; Leuven: Peeters 1993).

Feder, F., Biblia Sahidica. Ieremias, Lamentationes (Threni), Epistula Ieremiae et Baruch (Texte und Untersuchungen zur Geschichte der altchristlichen Literatur 147; Berlin - New York: De Gruyter 2002).

Hebbelynck, A., "Les manuscrits coptes-sahidiques du «Monastère Blanc». Recherches sur les fragments complémentaires de la Collection Borgia. I. Fragments de l'Ancien Testament," Muséon 12 (1911) 1-64.

Hebbelynck, A., "Fragments inédits de la version sahidique d'Isaïe. I. Fragments de la Bibliothèque Nationale de Paris," Muséon 14 (1913) 177-227.

Hurtado, L.W., The Earliest Christian Artifacts. Manuscripts and Christian Origins (Grand Rapids, MI: Eerdmans 2006).

Hyvernat, H., "Étude sur les versions coptes de la Bible. II. - Ce qui nous est parvenu des versions égyptiennes," Revue Biblique 5 (1896) 540-569.

Lacau, P., “Textes de l'Ancien Testament en copte sahidique," Recueil de Travaux Relatifs à la Philologie et à l'Archéologie Égyptiennes et Assyriennes. Nouvelle Série 7 (1901) 103-124.

Layton, B., A Coptic Grammar. With Chrestomathy and Glossary. Sahidic Dialect. Second Edition, Revised and Expanded. With an Index of Citations (Wiesbaden: Harrassowitz 2004).

Lust, J. - Eynikel, E. - Hauspie, K., Greek-English Lexicon of the Septuagint (Stuttgart: Deutsche Bibelgesellschaft 2003).

Maspero, G., "Fragments de manuscrits coptes-thébains provenant de la bibliothèque du Deir Amba-Shenoudah," Mémoires publiés par les membres de la Mission Archéologique Française au Caire 6 (1892) 1-296.

Nagel, P., "Studien zur Textüberlieferung des sahidischen Alten Testaments, Teil IB," Zeitschrift für Ägyptische Sprache und Altertumskunde 111 (1984) 138-164.

Nagel, P., "Editionen koptischer Bibeltexte seit Till 1960," Archiv für Papyrusforschung 35 (1990) 43-100.

Pietersma, A. -Wright, B.G. (eds.) A New English Translation of the Septuagint (New York: Oxford University Press 2007) (= NETS).

Porcher, M.E., "Analyse des manuscrits coptes $131^{1-8}$ de la Bibliothèque Nationale, avec indication des textes bibliques," Revue d'égyptologie 2 (1936) 65-123.

Rahlfs, A., Die alttestamentlichen Lektionen der griechischen Kirche (Mitteilungen des Septuaginta-Unternehmens der Königlichen Gesellschaft der Wissenschaften zu Göttingen 5; Berlin: Weidmann 1915). 


\section{A CRITICAL EDITION AND PHILOLOGICAL ANALYSISOF THE TEXT OF ISA 44:6-45:25}

Schleifer, J., Sahidische Bibel-Fragmente aus dem British Museum zu London (Sitzungsberichte der Kaiserlichen Akademie der Wissenschaften in Wien. Philosophisch-historische Klasse 162/6; Wien: Hölder 1909).

Schmitz, F.J. - Mink, G., Liste der koptischen Handschriften des Neuen Testaments. I. Die sahidischen Handschriften der Evangelien (Arbeiten zur Neutestamentlichen Textforschung 15; Berlin - New York: De Gruyter 1991) 2/2.

Schüssler, K., Das sahidische Alte und Neue Testament: sa 21-48 (Biblia Coptica 1/2; Wiesbaden: Harrassowitz 1996).

Schüssler, K., Das sahidische Alte und Neue Testament: sa 93-120 (Biblia Coptica 1/4; Wiesbaden: Harrassowitz 2000).

Schüssler, K., Das sahidische Alte und Neue Testament: sa 185-260 (Biblia Coptica 2/2; Wiesbaden: Harrassowitz 2015).

Takla, H.N., "The Surviving Remains of the Book of Jeremiah from Saint Shenouda's Monastery," Coptica 9 (2010) 83-89.

Till, W.C., "Die Coptica der Wiener Papyrussammlung," Zeitschrift der Deutschen Morgenländischen Gesellschaft 95 (1941) 165-218.

Till, W.C., "Papyrussammlung der Nationalbibliothek in Wien. Katalog der koptischen Bibelbruchstücke. Die Pergamente," Zeitschrift für die neutestamentliche Wissenschaft 39 (1940) 1-57.

Till, W.C., "La séparation des mots en copte," Bulletin de l'Institut Français d’Archéologie Orientale 60 (1960) 151-170.

Vaschalde, A., "Ce qui a été publié des versions coptes de la Bible," Revue Biblique 16 (1919) 220-243.

Vaschalde, A., "Ce qui a été publié des versions coptes de la Bible," Revue Biblique 29 (1920) 241-258.

Wessely, C., Griechische und koptische Texte theologischen Inhalts (Studien zur Palaeographie und Papyruskunde 15; Leipzig: Haessel 1914) IV.

Winstedt, E.O., "Some Unpublished Sahidic Fragments of the Old Testament," The Journal of Theological Studies 10 (1909) 233-254.

Ziegler, J. (ed.), Septuaginta. Vetus Testamentum Graecum. Auctoritate Societatis Litterarum Gottingensis editum. XIV. Isaias (Göttingen: Vandenhoeck \& Ruprecht 1939).

Zoega, G., Catalogus codicum copticorum manu scriptorum qui in museo Borgiano velitris adservantur (Roma: Typis Sacrae Congregationis de Propaganda Fide 1810). 
